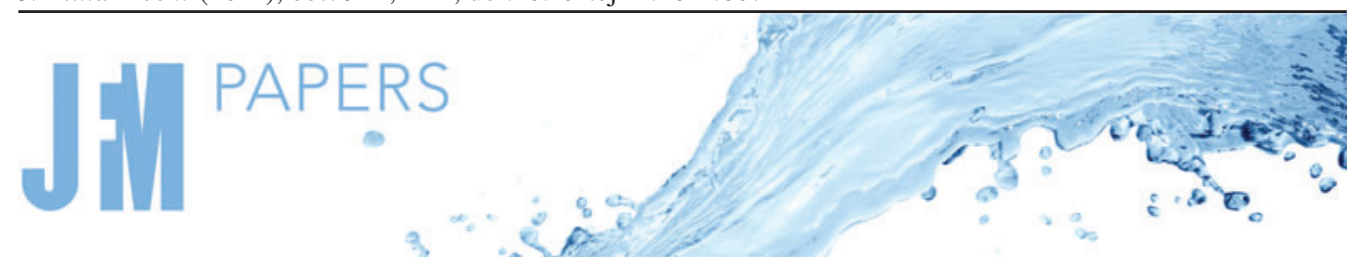

\title{
The relation between wave asymmetry and particle orbits analysed by Slepian models
}

\author{
Georg Lindgren ${ }^{1, \dagger}$ and Marc Prevosto ${ }^{2}$ \\ ${ }^{1}$ Mathematical Statistics, Lund University, 22100 Lund, Sweden \\ ${ }^{2}$ Independent researcher, Brest 29200, France
}

(Received 24 March 2021; revised 6 June 2021; accepted 1 July 2021)

The statistical relation between ocean wave geometry and water particle movements can be formulated in the stochastic Gauss-Lagrange model. In this paper we use Slepian models to obtain detailed information of the sea surface elevation in the neighbourhood of local maxima in a Gaussian wave model and of the movements of the top particle of the waves. We present full conditional distributions of the Gaussian vertical and horizontal movements in the Gauss-Lagrange model, and represent them as one regression component depending on the height and curvature at the wave maxima and one residual component. These conditional distributions define the explicit vertical and horizontal Slepian models. The Slepian models are used to simulate individual min-max-min waves in space, in particular their front-back asymmetry, and the velocity vector of the particle at the wave maximum. We find that there is a strong relation between the degree of front-back wave asymmetry and the direction of the particle movement. We discuss the role of second-order corrections to the Gaussian components and find only minor effects for the sea states studied. The Slepian model is shown to be an efficient tool to obtain detailed information about Gaussian and related models in the neighbourhood of critical points, without the need for time and space consuming simulations. In particular, they permit easy simulation of shape and kinematics of rare extreme waves.

Key words: computational methods, coastal engineering

\section{Introduction}

In an irregular sea there is a complex relation between the random geometry of the individual waves, measured by their front-back asymmetry, and the water particle orbits.

$\dagger$ Email address for correspondence: georg.lindgren@matstat.lu.se

(C) The Author(s), 2021. Published by Cambridge University Press. This is an Open Access article, distributed under the terms of the Creative Commons Attribution licence (http://creativecommons.org/ licenses/by/4.0/), which permits unrestricted re-use, distribution, and reproduction in any medium, provided the original work is properly cited. 


\section{G. Lindgren and M. Prevosto}

There are very few detailed observations of the relation between orbit shape and wave shape in the open ocean, and most studies are combinations of theoretical models, wave tank experiments and field data. For example, Chen, Hsu \& Chen (2010) and Chen et al. (2012) analyse theoretical models for monochromatic waves over uniform and sloping bottoms and compare particle orbits with experimental results. Grue \& Jensen (2012) and Grue \& Kolaas (2017) report particle velocities for both laboratory waves and directional ocean waves based on data from Romero \& Melville (2010) and reconstruct the orbits with respect to different phases and vertical positions. Nouguier, Guérin \& Chapron (2009), Nouguier, Chapron \& Guérin (2015) and Guérin et al. (2019) elaborate on the Gauss-Lagrange model to study semi-regular and irregular waves with consequences for particle orbit studies. van den Bremer et al. (2019) and Calvert et al. (2019) make detailed models for depth dependent particle drifts in very regular wave packets and compare numerical results with laboratory studies on set-down, particle orbits and Lagrangian displacement.

The relations between the front-back asymmetry of individual waves in space and time and the geometry of particle orbits were recently studied by Monte Carlo simulations of the Gaussian components in the Gauss-Lagrange wave model (Lindgren \& Prevosto 2020). In this paper, we will use an alternative technique, where one can simulate individual waves centred at local maxima with random or predetermined height without having to generate long time or space series. The method, named Slepian models after David Slepian (Slepian 1963), is statistically based on the conditional distributions of the wave components given the occurrence of a local wave maximum.

The Gauss-Lagrange wave model for irregular ocean waves was proposed by Pierson (1961) as an explicit means to include particle kinematics in a stochastic wave model. It describes the vertical and horizontal movements of each particle on the sea surface as two correlated Gaussian random processes, one for the particle vertical movement and one for its horizontal displacement from an assumed original location. A Slepian model for wave shape and orbit around a local wave maximum gives the conditional distributions of the Gaussian components of the Lagrange model, conditional on the presence of a zero crossing in the vertical process derivative with negative second derivative. The model gives an explicit representation of the involved variables and it is easy to simulate and much less time consuming than time series generation, and its explicit form gives additional information of the wave properties.

The Slepian model is a versatile statistical tool with many applications, including simulation of extreme events, asymptotic analysis and approximation of complex stochastic structures. The original model in Slepian (1963) was extended in Lindgren $(1970,1972)$ to models for the wave shape near a local maximum in a Gaussian wave in time and two-dimensional space, respectively. Using the term 'model field' Adler (1981, Chapter 6) expands the theory to an $N$-dimensional homogeneous Gaussian field and analyses in detail the behaviour near high maxima. The term Slepian model was introduced in Lindgren (1977) and is now widely accepted (Blanco-Pillado, Sousa \& Urkiola 2020).

The Slepian model has found extensive use in ocean science and engineering. The basic Slepian models were applied to Gaussian ocean waves in Lindgren \& Rychlik (1982), Rychlik (1987) and Lindgren \& Rychlik (1991) to find the joint distribution of the period and amplitude of Gaussian waves. Tromans, Anaturk \& Hagemeijer (1991) refer to the Slepian model and introduce the term New Wave theory for the extreme case of a high crest applied to design waves. Winterstein, Torhaug \& Kumar (1998) used the Slepian model to find design sea states for extreme response of jackup structures, while an application to buoy response for wave data acquisition is found in Niedzwecki \& Sandt (1999). 
Boccotti (1982, 1983) made numerical experiments with a simplified Slepian model and illustrated graphically the variability of the conditional distribution. Boccotti (1984) later developed a theory of 'quasi-determinism' for Slepian models for very high waves, (Boccotti 2000, 2015). Phillips, Gu \& Donelan (1993a) and Phillips, Gu \& Walsh (1993b) made more detailed studies of the conditional model for high waves and compared with many different datasets. Whittaker et al. (2016) also compared the theoretical residual variance from Lindgren (1970) with observed high waves and found excellent agreement. Dimentberg, Iourtchenko \& Naess (2006) used the Slepian model as a ship design tool to study instability.

A more recent ocean application of the Slepian model is to Gauss-Lagrange waves, where both vertical and horizontal movements are modelled as Gaussian processes (Lindgren 2006) with a correction in Åberg (2007, Appendix B) and Lindgren (2015). DiBenedetto (2020) use a 'wave-phase variability' that has some resemblance to the Slepian model to investigate the spread and distribution of buoyant particles in the ocean, emphasising pollution effects. Hlophe et al. (2021) make extensive use of crossing conditioning for wave-to-wave prediction of wave fields. The purpose of the present paper is to describe and further illustrate the use of Slepian models in the Gauss-Lagrange setting and draw conclusions pertaining to the relation between wave geometry and orbit geometry.

Examples of Slepian models in statistical theory are Gadrich \& Adler (1993) on non-stationary processes, Baxevani \& Wilson (2018) on prediction of extreme events in space over time, Baxevani, Podgórski \& Rychlik (2003) on velocities of random surfaces, Azaïs \& Chassan (2020) on statistical extreme value theory and Podgórski, Rychlik \& Wallin (2015) on the Laplace moving average.

Advanced applications of the Slepian model are found in signal processing (Szajnowski 1996) for bandwidth estimation, in seismic design to describe plastic deformation (Lazarov \& Ditlevsen 2005; Feau 2008), optics to describe an optical vortex, also called a 'black hole', (Lindgren 2012), cosmology to describe high minima and low maxima in energy fields (Blanco-Pillado et al. 2020) and frequently over many years in safety studies in mechanical and structural engineering by Ditlevsen (1985) and subsequently Sobczyk (1993, §§ 4.4-4.5), Randrup-Thomsen \& Ditlevsen (1997), Ditlevsen \& Tarp-Johansen (1999), van de Lindt \& Niedzwecki (2005) and Grigoriu (2020).

In this paper we use the power of the Slepian model to give precise analytical as well as experimental details on selected events in a time/space series or random field, even when these events are very rare. In $\S 2$ we describe the Gauss-Lagrange wave model to motivate the need for separate Slepian models for water particle movements in space and time. In $\S 3$ we motivate the Slepian model and describe its interpretation as a long-run distribution. Section 4 gives a detailed description of the Slepian models in the one-dimensional Gaussian case. Section 5 presents the four-dimensional Slepian model in space and time for the vertical and horizontal components in the Gauss-Lagrange wave model, with numerical illustrations in $\$ 6$ for the Gaussian components and in $\S 7$ for the resulting Lagrange waves. In $\S 8$ we discuss further aspects of our approach, with a summary in $\S 9$.

Before we proceed, we clarify and motivate the terminology used in this paper. The Gauss-Lagrange model, (Pierson 1961), describes explicitly the vertical and horizontal movements of individual water particles as two dependent Gaussian fields, asymptotically, when $N \rightarrow \infty$, as (2.2) and (2.1), respectively. The dependence is determined by the depth and frequency dependent hydrodynamic Miche/Gerstner (Gerstner 1809; Miche 1944) relations with no interaction between frequencies. It is intrinsically a linear model. 


\section{G. Lindgren and M. Prevosto}

Gauss-Lagrange waves or, for short, Lagrange waves are the combined results of the Gaussian components in the Gauss-Lagrange model, as defined by (2.3) and (2.4). Interaction between frequencies causes implicit effects similar to what are found in secondand higher-order Stokes models (Tayfun 1980). Explicit Stokes effects can be added to the Gaussian components in the Gauss-Lagrange model, resulting in a Stokes-Lagrange model; see Lindgren \& Prevosto (2020). Regardless of how interaction is introduced, the effect on local wave characteristic is small, at least on deep water.

\section{The Gauss-Lagrange wave model}

The two-dimensional Gauss-Lagrange wave model consists of two correlated stationary and homogeneous Gaussian random fields, $W(u, t)$ and $X(u, t)$, where $u$ is a one-dimensional space parameter and $t$ is the time parameter. The pair $(W(u, t), u+$ $X(u, t))$ represents the vertical and horizontal position at time $t$ of a water particle at the surface, originally located at position $u$, with $W(u, t)$ the vertical distance from the still water level, and $X(u, t)$ the horizontal displacement from the particle origin. Together, the fields define the orbital movements of the water particles as functions of time. We consider here only particles at the free surface but the model extends to general depth.

Following Pierson (1961), the energy spectrum $S(\omega)$ of the vertical field is called the orbital spectrum. It is not identical to the Euler spectrum, obtained from observations of the ocean surface, but the difference is of no relevance in the present work. Representing the continuous energy spectrum by a discrete spectrum over frequencies $\omega_{j}=j \Delta \omega$ and wavenumbers $\kappa_{j}$, we write the models for particles on the free surface as

$$
\begin{gathered}
\text { Vertical position : } W(u, t)=\sum_{j} A_{j} \cos \left(\kappa_{j} u-\omega_{j} t-\phi_{j}\right), \\
\text { Horizontal displacement : } X(u, t)=\sum_{j} A_{j} h_{j} \cos \left(\kappa_{j} u-\omega_{j} t-\phi_{j}+\pi / 2\right) .
\end{gathered}
$$

Here, $h_{j}=1 / \tanh \left(\kappa_{j} h\right)=\cosh \left(\kappa_{j} h\right) / \sinh \left(\kappa_{j} h\right)$ is the depth dependent amplitude gain factor, with dispersion relation $\omega_{j}=\sqrt{g \kappa_{j} \tanh \kappa_{j} h}$, where $g$ is the constant of gravitation. We assume $\kappa_{j}>0$ and $\omega_{j}>0$ so waves are unidirectional, moving from left to right.

The amplitudes $A_{j}$ are random, $A_{j}=\sqrt{a_{j}^{2}+b_{j}^{2}}$, with independent normal variables $a_{j}, b_{j}$, with mean zero and equal variance such that $A_{j}^{2}$ has expected value $2 S\left(\omega_{j}\right) \Delta \omega$. The relative phases $\phi_{j}$ are independent and uniformly distributed in $[0,2 \pi]$. The phase shift between vertical and horizontal movements is $\pi / 2$ as in (2.2), independent of frequency.

From the pair $W(u, t), X(u, t)$ one can implicitly define a Lagrange wave $L(x, t)$ by

$$
L(u+X(u, t), t)=W(u, t),
$$

that is, the sea surface at time $t$ at location $x=u+X(u, t)$ is $W(u, t)$. Keeping time fixed $t=t_{0}$, we get a space wave,

$$
L\left(x, t_{0}\right)=L\left(u+X\left(u, t_{0}\right), t_{0}\right)=W\left(u, t_{0}\right),
$$

as a continuous parametric curve in space. The curve may be multiple valued unless $u \mapsto$ $u+X\left(u, t_{0}\right)$ is strictly increasing. Since $X\left(u, t_{0}\right)$ is continuous there is a unique relation between local maxima of the Gaussian process $W\left(u, t_{0}\right)$ and the Lagrange space wave 


\section{Orbit orientation in asymmetric waves}

$L\left(x, t_{0}\right)$. Keeping the space coordinate fixed $x=x_{0}$, we get a time wave, satisfying

$$
L\left(x_{0}, t\right)=W\left(u_{t}, t\right), \quad u_{t}+X\left(u_{t}, t\right)=x_{0} .
$$

Again, if $u \mapsto u+X\left(u, t_{0}\right)$ is strictly increasing for each $t$ the Lagrange time wave is uniquely defined by $(2.5 a, b)$. (Since the derivative $\partial X(u, t) / \partial u$ is Gaussian and stationary there is a positive probability that the function is not strictly increasing. For normal ocean spectra over moderate sized regions the probability of this happening is very small.)

\section{Interpretation and integral form of Slepian models at wave maxima}

\subsection{Counting maxima and marked maxima}

A Slepian model is a stochastic model of any dimension or complexity that represents the distribution of a group of variables or processes conditioned on a crossing event; (Leadbetter, Lindgren \& Rootzén 1983, chapter 10.3). These variables are called marks, attached to the crossings. In our case, the crossings will be the local maxima of the space wave $W\left(u, t_{0}\right)$ or $L\left(x, t_{0}\right)$. Examples of simple marks are the height of the maximum and the horizontal and vertical velocities of the water particle at the maximum at the time of observation. More complex marks are the wave shape in the vicinity of the maximum and the time orbit of the water particle that was located at the maximum. In the main text we use the local min-max-min definition of a wave. The trough-crest-trough definition is discussed in $\S 7.3$.

We start with the Slepian models for the individual Gaussian components. We suppress the constant $t_{0}$ in the rest of this section and write $W$ and $W_{u}, W_{u u}$, the first and second partial derivatives, without $u$-argument, when it is clear from the context if they represent general values or conditional values at maxima. For the velocities we write $W_{t}$ for the vertical and $X_{t}$ for the horizontal velocity of the maximum particle.

To make a frequentist's definition of the Slepian model and its distribution we count the total number of local maxima of $W(u)$ in a space interval $0 \leqslant u \leqslant U$ (even if $u+X(u)$ is not strictly increasing the local derivative has a zero crossing at the maximum),

$$
N_{U}=\#\left\{u_{k} \in[0, U] ; W\left(u_{k}\right) \text { is a local maximum }\right\},
$$

and identify those marked maxima where $W\left(u_{k}+\cdot, \cdot\right)$ and $X\left(u_{k}+\cdot, \cdot\right)$ jointly satisfy some well-defined condition $\mathcal{A}$,

$$
N_{U}(\mathcal{A})=\#\left\{u_{k} \in[0, U] ; W\left(u_{k}\right) \text { is a local maximum where condition } \mathcal{A} \text { is satisfied }\right\},
$$

with $N_{U}(\mathcal{A}) / N_{U}$ giving the relative frequency of maxima where condition $\mathcal{A}$ is satisfied.

As examples of one-dimensional conditions $\mathcal{A}$ we can take

$$
\left\{W\left(u_{k}\right) \leqslant w\right\},\left\{W_{t}\left(u_{k}\right) \leqslant v\right\},\left\{W\left(u_{k}+s^{\prime}, t^{\prime}\right) \leqslant y^{\prime}\right\},\left\{X\left(u_{k}+s^{\prime \prime}, t^{\prime \prime}\right) \leqslant y^{\prime \prime}\right\},
$$

while $\left\{W_{t}\left(u_{k}\right) \leqslant v_{v} \& X_{t}\left(u_{k}\right) \leqslant v_{h}\right\}$ is a simple example of a bi-variate condition, that we will investigate later. Taking higher-dimensional conditions we can get the full distribution of the $W$ - and $X$-components near a wave maximum in space and we will do so in $\S 5$.

When the $W, X$-system is ergodic, which is the case when the orbital spectrum is continuous, the empirical distribution converges as $U \rightarrow \infty$,

$$
\lim _{U \rightarrow \infty} \frac{N_{U}(\mathcal{A})}{N_{U}}=\frac{E\left(N_{1}(\mathcal{A})\right)}{E\left(N_{1}\right)}=Q(\mathcal{A}), \text { say. }
$$

The interpretation of $Q(\mathcal{A})$ is as the long-run distribution of the $W$ - and $X$-fields in the neighbourhood of local space maxima. 


\section{G. Lindgren and M. Prevosto}

3.2. The expectations in $Q(\mathcal{A})$

To get an explicit representation of the distribution we give integral expressions for the expectations in (3.4). The denominator $E\left(N_{1}\right)$ is the mean number of maxima per space unit, by Rice's formula equal to

$$
E\left(W_{u u}(0)^{-} \mid W_{u}(0)=0\right) f_{W_{u}}(0)=f_{W_{u}}(0) \int_{z=-\infty}^{0}(-z) f_{W_{u u} \mid W_{u}=0}(z) \mathrm{d} z,
$$

where $f_{W_{u}}$ is the probability density function of $W_{u}(0)$ and $f_{W_{u u} \mid W_{u}=0}(z)$ a conditional density.

To get compact notation we define $W_{0, u, u u}=(a, b, c)$ as $W(0)=a, W_{u}(0)=$ $b, W_{u u}(0)=c$ and $W_{u, u u}=(b, c)$ as $W_{u}(0)=b, W_{u u}(0)=c$, etc. Then the nominator in (3.4) is expressed by a generalised Rice's formula as

$$
\begin{aligned}
E\left(N_{1}(\mathcal{A})\right) & =E\left(W_{u u}^{-} I_{\mathcal{A}} \mid W_{u}=0\right) f_{W_{u}}(0) \\
& =f_{W_{u}}(0) \int_{-\infty}^{0} E\left(I_{\mathcal{A}} \mid W_{u, u u}(0, z)\right)(-z) f_{W_{u u} \mid W_{u}=0}(z) \mathrm{d} z .
\end{aligned}
$$

The indicator function $I_{\mathcal{A}}=I_{\mathcal{A}}(W, X)$ is equal to one if the $W$ - and $X$-functions satisfy the condition $\mathcal{A}$.

\subsubsection{Extended conditioning at maxima}

To get maximal use of the Slepian model one can engage also the height of the maximum, $W\left(u_{k}\right)$, in the model, when we express $E\left(N_{1}(\mathcal{A})\right)$ in integral form. Then

$$
\begin{aligned}
E\left(N_{1}(\mathcal{A})\right)= & f_{W_{u}}(0) \\
& \times \int_{a} \int_{z=-\infty}^{0} E\left(I_{\mathcal{A}} \mid W_{0, u, u u}=(a, 0, z)\right)(-z) f_{W, W_{u u} \mid W_{u}=0}(a, z) \mathrm{d} z \mathrm{~d} a .
\end{aligned}
$$

From (3.7) we get the joint density of the height $W$ and second derivative $W_{u u}$ at local space maxima,

$$
q(z, a)=\frac{(-z) f_{W, W_{u u} \mid W_{u}=0}(a, z)}{\int_{a} \int_{z=-\infty}^{0}(-z) f_{W, W_{u u} \mid W_{u}=0}(a, z) \mathrm{d} z \mathrm{~d} a}, \quad z<0,-\infty<a<\infty,
$$

and we can express the expectation in (3.4) as

$$
E\left(N_{1}(\mathcal{A})\right)=\int_{a} \int_{z=-\infty}^{0} E\left(I_{\mathcal{A}}(W, X) \mid W_{0, u, u u}=(a, 0, z)\right) q(z, a) \mathrm{d} z \mathrm{~d} a .
$$

\section{Local structure in the Gaussian case}

The Gauss and the Gauss-Lagrange wave models are completely defined by the one-sided spectral density $S(\omega)$ for the stationary time process $W\left(u_{0}, t\right)$, in the Gauss-Lagrange model called the orbital spectrum. From $S(\omega)$ one can compute time and space covariance functions as well as cross-covariance functions for the $W$ - and $X$-processes. 


$\begin{array}{lccc}\text { Variable } & E\left(\cdot \mid W_{u, u u}(0, z)\right) & V\left(\cdot \mid W_{u, u u}(0, z)\right) & \operatorname{Cov}\left(\cdot, \cdot \mid W_{u, u u}(0, z)\right) \\ X_{t} & -\hat{m}_{12} z / m_{04} & \Delta_{x_{t}}=\hat{m}_{20}-\hat{m}_{12}^{2} / m_{04} & - \\ X_{t}, W & - & - & \Delta_{w, x_{t}}=\hat{m}_{10}-m_{02} \hat{m}_{12} / m_{04} \\ W & -m_{02} z / m_{04} & \Delta_{w}=m_{0}-m_{02}^{2} / m_{04} & - \\ W_{t} & 0 & \Delta_{w_{t}}=m_{20}-m_{11}^{2} / m_{02} & -\end{array}$

Table 1. Conditional moments at local maximum.

We use the following standard notation for covariance functions and covariances. For the covariance functions we write

$$
\left.\begin{array}{rl}
r^{w x}(u, t) & =\operatorname{Cov}(W(0,0), X(u, t)), \\
r^{w_{u} x}(u, t) & =\operatorname{Cov}\left(W_{u}(0,0), X(u, t)\right), \\
r^{x_{t} w_{u u}}(u, t) & =\operatorname{Cov}\left(X_{t}(0,0), W_{u u}(u, t)\right),
\end{array}\right\}
$$

etc. We reserve the notation $r(t)=\int \cos (\omega t) S(\omega) \mathrm{d} \omega$ for the time covariance function of $W\left(u_{0}, t\right)$ and let $m_{0}=V(W(u, t))$ be the variance. For the covariances we use the standard spectral moments $m_{i j}=\int \omega^{i} \kappa^{j} S(\omega) \mathrm{d} \omega$ when only the $W$-field is involved. For the $X$-field and for mixed $W, X$ moments we use a 'hat'-notation when needed,

$$
\left.\begin{array}{c}
\hat{m}_{0}=\int \rho^{2} S(\omega) \mathrm{d} \omega, \quad \hat{m}_{10}=\int \rho \omega S(\omega) \mathrm{d} \omega, \\
\hat{m}_{20}=\int \rho^{2} \omega^{2} S(\omega) \mathrm{d} \omega, \quad \hat{m}_{12}=\int \rho \omega \kappa^{2} S(\omega) \mathrm{d} \omega,
\end{array}\right\}
$$

with $\rho=1 / \tanh (h \kappa)$; see Appendix A for a listing of notation.

\subsection{Statistical properties at a local maximum}

For the Gaussian case we start with explicit expressions for a few simple and important variables coupled to local maxima, namely the maximum height and the vertical and horizontal velocities of the particle located at the maximum at time of observation, i.e. $W=W\left(u_{k}\right), W_{t}=W_{t}\left(u_{k}\right), X_{t}=X_{t}\left(u_{k}\right)$.

They can all be expressed via formula (3.6), translated to probability densities for the three cases. The unconditional distribution of $\left(X_{t}, W, W_{u u}, W_{u}, W_{t}\right)$ is normal with zero mean and covariance matrix

$$
\Sigma=\left[\begin{array}{ccccc}
\hat{m}_{20} & \hat{m}_{10} & -\hat{m}_{12} & 0 & 0 \\
\hat{m}_{10} & m_{0} & -m_{02} & 0 & 0 \\
-\hat{m}_{12} & -m_{02} & m_{04} & 0 & 0 \\
0 & 0 & 0 & m_{02} & -m_{11} \\
0 & 0 & 0 & -m_{11} & m_{20}
\end{array}\right]
$$

From the covariance matrix we draw the conclusion that, of the two characteristic variables at a local maximum, $W_{u}=0$ and $W_{u u}=z$, the former affects only $W_{t}$ and the latter affects only $X_{t}, W$. The conditional distribution of $\left(X_{t}, W, W_{t}\right)$ given $W_{u, u u}=(0, z)$ as normal with mean, variances and covariance as in table 1 . This leads to the following simple representations of the involved variables.

Since the factor $(-z) f_{W_{u u} \mid W_{u}=0}(z)$ in (3.6) is proportional to a negative Rayleigh density with parameter $\sqrt{m_{04}}$ the Rice formula (3.6) leads to the explicit representation (4.5) of 


\section{G. Lindgren and M. Prevosto}

the three variables at a local space maximum. Let $N^{w_{t}}$ be a standard normal variable and let $\left(N^{w}, N^{x_{t}}\right)$ be an independent pair of standard normal variables with correlation coefficient $\Delta_{w, x_{t}} / \sqrt{\Delta_{w} \Delta_{x_{t}}}$. Also let $R$ be a standard Rayleigh variable, independent of the normals. Then, at a local maximum, with $={ }^{\mathcal{L}}$ denoting 'equal in distribution', the curvature distribution is expressed as

$$
W_{u u}={ }^{\mathcal{L}} Z=-\sqrt{m_{04}} R
$$

while the remaining distributions can be expressed as

$$
\left.\begin{array}{c}
W={ }^{\mathcal{L}} \sqrt{\Delta_{w}} N^{w}+m_{02} R / \sqrt{m_{04}}, \\
W_{t}={ }^{\mathcal{L}} \sqrt{\Delta_{w_{t}}} N^{w_{t}}, \\
X_{t}={ }^{\mathcal{L}} \sqrt{\Delta_{x_{t}}} N^{x_{t}}+\hat{m}_{12} R / \sqrt{m_{04}} .
\end{array}\right\}
$$

Note that $W$ and $X_{t}$ are dependent both through the common $R$ and through the normal correlation, while $W_{t}$ is independent of the two. In fact, the three expressions in (4.5) together with the definition (4.4) is a rudimentary example of a Slepian model, with a crossing-defined regression term and Gaussian residuals.

\subsection{Particle velocities}

We will use (4.4)-(4.5) to find the distribution of particle velocities at the wave maximum and its dependence of the maximum height.

Obviously, the vertical velocity $W_{t}$ is normal with mean zero and variance $\Delta_{w_{t}}$. The marginal distribution of $W$ and $X_{t}$ is that of the sum of a normal and a Rayleigh variable and it was derived by Rice $(1945, \S 3.6)$ as the distribution of the local maxima of a Gaussian process, i.e. the representation of $W$. A general form of the probability density function (p.d.f.) is given in Appendix B, (B1); see also Prevosto (2020).

The joint distribution of $W, X_{t}$ is a bivariate normal distribution shifted by a Rayleigh distributed vector. A derivation of the p.d.f. is given in Appendix B, Fact B.2.

To illustrate the results, we consider the joint distribution of vertical and horizontal velocities and how it varies with the maximum height. Let $f_{W_{t}}(v)$ be the normal density of vertical velocity and let $f_{W, X_{t}}(w, h)$ be the joint density of height and horizontal velocity. The combined density is

$$
f_{W, X_{t}, W_{t}}(w, h, v)=f_{W, X_{t}}(w, h) \times f_{W_{t}}(v),
$$

and the conditional density, with $c$ as a normalising constant,

$$
f_{X_{t}, W_{t} \mid W=w_{0}}(h, v)=c f_{W, X_{t}}\left(w_{0}, h\right) \times f_{W_{t}}(v) .
$$

Figure 1 shows how the joint distribution depends on the height of the maximum for a JONSWAP wave spectrum J20 described in $\S 6$. In panel $(a)$, calculated by (4.7), the wave height is exactly $w_{0}$. In panel $(b)$, the density (4.6) is integrated over $w>w_{0}$ to get all waves greater than $w_{0}$. In figure 2 the constraint of the maximum is absent. The figure shows the joint p.d.f. from (B1) together with an empirical p.d.f. based on 100000 independent Slepian realisations. 


\section{Orbit orientation in asymmetric waves}

(a) $\quad h / v$ velocity pdf depending on wave height

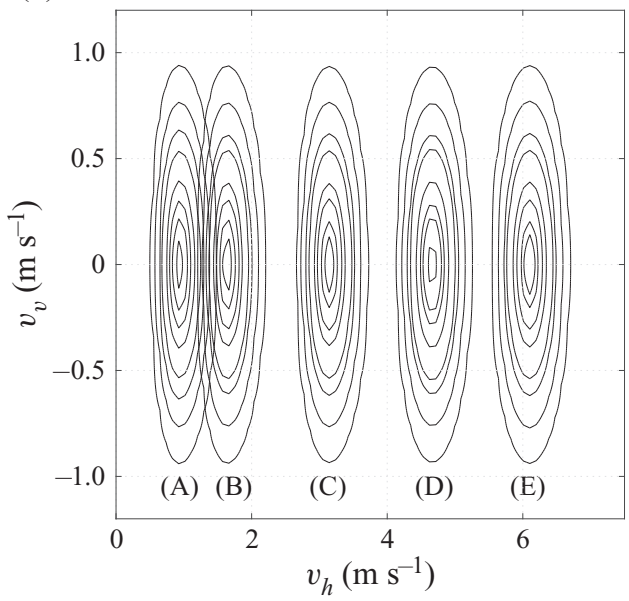

(b) Joint vertical and horizontal velocity pdf

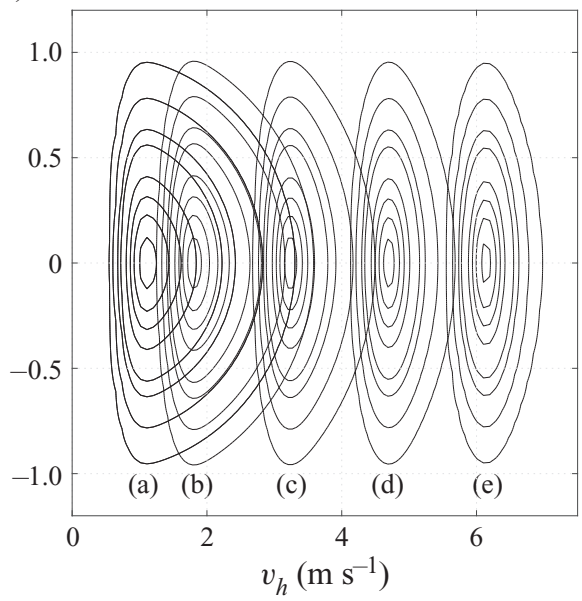

Figure 1. Joint horizontal/vertical velocity depending on wave height for a J20 spectrum. (a) At wave max equal to $w_{0}$. (b) At wave max exceeding $w_{0}$. (A/a)-(E/e): $w_{0}=\left[\begin{array}{lllll}0.25 & 0.5 & 1 & 1.5 & 2\end{array}\right] \times H_{s}$. The level curves enclose $10: 20: 90,95,99,99.9 \%$ of the distribution.

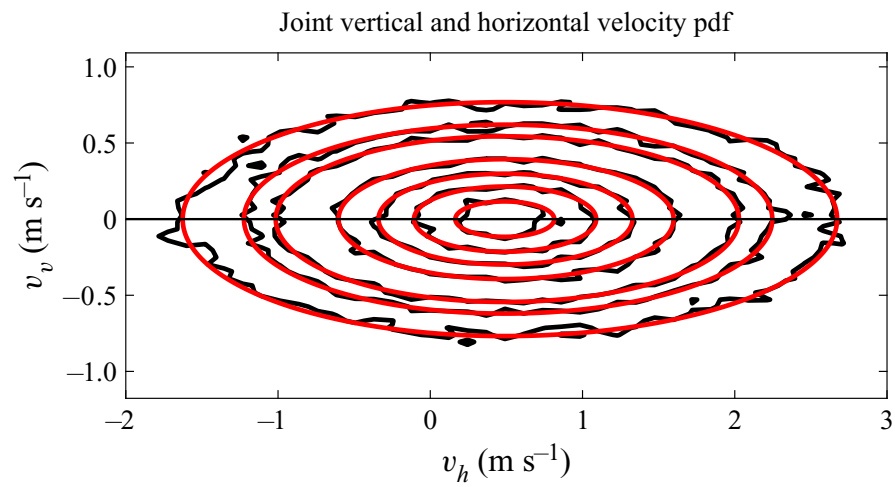

Figure 2. Joint density of $v_{h}, v_{v}$, horizontal and vertical particle velocities at local maxima from (B1) (smooth, red curves) together with empirical p.d.f. from 100000 simulated Slepian realisations (wiggly black curves). Level curves as in figure 1. The wave spectrum is J20 at infinite depth. The level curves enclose $10: 20: 90,95$, $99 \%$ of the distribution.

\subsection{Interpretation of the q-distribution (3.8) in the Gaussian case}

In the following sections we will write $A=W$ and $Z=W_{u u}$ for the random height and curvature of the maximum and express their joint density function (3.8) in explicit form,

$$
q(z, a)=\frac{-z}{k} \exp \left\{-\frac{m_{0} z^{2}+2 m_{02} a z+m_{4} a^{2}}{2\left(m_{0} m_{04}-m_{02}^{2}\right)}\right\}, \quad z<0,
$$

where $k$ is a generic normalising constant. The following forms of the $q$-density are useful for simulation purposes:

$$
q(z, a)=\frac{-z}{k} \exp \left\{-\frac{z^{2}}{2 m_{04}}\right\} \times \exp \left\{-\frac{\left(a+z m_{02} / m_{04}\right)^{2}}{2\left(m_{0}-m_{02}^{2} / m_{04}\right)}\right\}, \quad z<0,
$$




\section{G. Lindgren and M. Prevosto}

$$
q(z \mid a)=\frac{-z}{k_{a}} \exp \left\{-\frac{\left(z+m_{02} a / m_{0}\right)^{2}}{2\left(m_{04}-m_{02}^{2} / m_{0}\right)}\right\}, \quad z<0,
$$

where $k, k_{a}$ are normalising constants.

The first form is equivalent to the representation of $W$ in (4.5) as the sum of a normal and a Rayleigh variable. The form (4.10) was used in Lindgren (1970) and it can be used to generate Slepian waves with fixed height $A=a$. We used simple rejection sampling by the MATLAB routine sampleDist to simulate from this non-standard distribution.

\subsection{Slepian models around wave crests}

The Slepian model around a local maximum is defined from local characteristics, and the conditional distributions are defined explicitly in the Gaussian context. A more 'regional' wave definition is the one centred around the wave crests, i.e. the maxima between two successive mean level crossings. Any corresponding wave definition, like the trough-crest-trough wave or the zero-crest-zero half-wave, is complex involving non-local conditions. However, since any wave crest is also a local maximum, simulation of crest waves is easily performed by means of local maxima Slepian waves. One simply rejects those samples which do not satisfy the crest wave definition. In $\S 7.3$ we will briefly investigate some of the complications and peculiarities that accompany this technique.

\section{Functional structure of the Slepian processes}

\subsection{The regression functions and the residual functions}

A Slepian model in a Gaussian model consists of one regression part with parameters determined by the random crossing event and one residual part for the variation around the regression. We base the models on representation (3.9), conditioning on the height $A=W$ and the curvature $Z=W_{u u}$ at a local maximum, where $W_{u}=0$.

The Slepian processes for the $W$ - and $X$-components in the Gauss-Lagrange model have the structures

$$
\begin{gathered}
\mathcal{W}(u, t)=A \alpha^{w}(u, t)+Z \beta^{w}(u, t)+\Delta^{w}(u, t), \\
\mathcal{X}(u, t)=A \alpha^{x}(u, t)+Z \beta^{x}(u, t)+\Delta^{x}(u . t),
\end{gathered}
$$

where the $\alpha$ - and $\beta$-functions are deterministic functions determined by the variances and covariances (A2) and (A3) and the $\Delta$-functions are non-stationary, mean zero, correlated Gaussian fields, independent of $A$ and $Z$, whose density is (4.8). The joint distribution of the processes $\mathcal{W}, \mathcal{X}$ in (5.1)-(5.2) is equal to the conditional distribution of $W, X$ around a local $W$-maximum in space. If we fix $A=a$ and generate $Z$ from (4.10) we get Slepian models near a wave with that height.

The next step is to describe the functions $\alpha$ and $\beta$ and the covariances for the $\Delta$-fields. The conditional distributions of $W(u, t), X(u, t)$ given the presence of a maximum at $\mathbf{0}=(0,0)$ are not normal. If we take the conditioning one step further and specify also the height and curvature at the maximum, the conditional distributions are again normal (Lindgren 1972) and determined by conditional expectations and covariances. Thus we need only consider these functions for a pair of space-time points, $\boldsymbol{p}_{j}=\left(u_{j}, t_{j}\right), j=1,2$, separated by $\boldsymbol{p}=\boldsymbol{p}_{2}-\boldsymbol{p}_{1}$. 
In short, set $W_{0}=\left(W(\mathbf{0}), W_{u}(\mathbf{0}), W_{u u}(\mathbf{0})\right)$. The $7 \times 7$ partitioned covariance matrix of $\left(W\left(p_{1}\right) W\left(p_{2}\right)\left|X\left(p_{1}\right) X\left(p_{2}\right)\right| W_{0}\right)=\left(W, X, W_{0}\right)$, is

$$
\begin{aligned}
\Sigma & =\left[\begin{array}{ccc}
\Sigma_{W W} & \Sigma_{W X} & \Sigma_{W W_{0}} \\
\Sigma_{X W} & \Sigma_{X X} & \Sigma_{X W_{0}} \\
\Sigma_{W_{0} W} & \Sigma_{W_{0} X} & \Sigma_{W_{0} W_{0}}
\end{array}\right] \\
& =\left(\begin{array}{cc|cc|ccc}
m_{0} & r^{w w}(\boldsymbol{p}) & r^{w x}(\mathbf{0}) & r^{w x}(\boldsymbol{p}) & r^{w w}\left(\boldsymbol{p}_{1}\right) & r^{w_{u} w}\left(\boldsymbol{p}_{1}\right) & r^{w_{u u} w}\left(\boldsymbol{p}_{1}\right) \\
r^{w w}(\boldsymbol{p}) & m_{0} & r^{w x}(-\boldsymbol{p}) & r^{w x}(\mathbf{0}) & r^{w w}\left(\boldsymbol{p}_{2}\right) & r^{w_{u} w}\left(\boldsymbol{p}_{2}\right) & r^{w_{u u} w}\left(\boldsymbol{p}_{2}\right) \\
\hline r^{w x}(\mathbf{0}) & r^{w x}(-\boldsymbol{p}) & \hat{m}_{0} & r^{x x}(\boldsymbol{p}) & r^{w x}\left(\boldsymbol{p}_{1}\right) & r^{w_{u} x}\left(\boldsymbol{p}_{1}\right) & r^{w_{u u} x}\left(\boldsymbol{p}_{1}\right) \\
r^{w x}(\boldsymbol{p}) & r^{w x}(\mathbf{0}) & r^{x x}\left(\boldsymbol{p}_{)}\right. & \hat{m}_{0} & r^{w x}\left(\boldsymbol{p}_{2}\right) & r^{w_{u} x}\left(\boldsymbol{p}_{2}\right) & r^{w_{u u} x}\left(\boldsymbol{p}_{2}\right) \\
\hline r^{w w}\left(\boldsymbol{p}_{1}\right) & r^{w w}\left(\boldsymbol{p}_{2}\right) & r^{w x}\left(\boldsymbol{p}_{1}\right) & r^{w x}\left(\boldsymbol{p}_{2}\right) & m_{0} & 0 & -m_{02} \\
r^{w_{u} w}\left(\boldsymbol{p}_{1}\right) & r^{w_{u} w}\left(\boldsymbol{p}_{2}\right) & r^{w_{u} x}\left(\boldsymbol{p}_{1}\right) & r^{w_{u} x}\left(\boldsymbol{p}_{2}\right) & 0 & m_{02} & 0 \\
r^{w_{u u} w}\left(\boldsymbol{p}_{1}\right) & r^{w_{u u} w}\left(\boldsymbol{p}_{2}\right) & r^{w_{u u} x}\left(\boldsymbol{p}_{1}\right) & r^{w_{u u} x}\left(\boldsymbol{p}_{2}\right) & -m_{02} & 0 & m_{04}
\end{array}\right) .
\end{aligned}
$$

The conditional joint distribution of the processes $W(u, t)$ and $X(u, t)$, given $W_{0}=$ $(a, 0, z)$, is normal with mean and covariance

$$
\begin{aligned}
& {\left[\begin{array}{l}
m_{w}(u, t) \\
m_{x}(u, t)
\end{array}\right]=\left[\begin{array}{lll}
r^{w w}(u, t) & r^{w_{u} w}(u, t) & r^{w_{u u} w}(u, t) \\
r^{w x}(u, t) & r^{w_{u} x}(u, t) & r^{w_{u u}}(u, t)
\end{array}\right] \Sigma_{W_{0} W_{0}}^{-1}\left[\begin{array}{l}
a \\
0 \\
z
\end{array}\right],} \\
& \operatorname{Cov}\left(W\left(p_{1}\right), W\left(p_{2}\right), X\left(p_{1}\right), X\left(p_{2}\right) \mid W_{0}\right) \\
& =\operatorname{Cov}\left(\Delta^{w}\left(p_{1}\right), \Delta^{w}\left(p_{2}\right), \Delta^{x}\left(p_{1}\right), \Delta^{x}\left(p_{2}\right)\right) \\
& =\left[\begin{array}{ll}
C_{\mathcal{W W}} & C_{\mathcal{W X}} \\
C_{\mathcal{X} \mathcal{W}} & C_{\mathcal{X X}}
\end{array}\right]=\left[\begin{array}{cc}
\Sigma_{W W} & \Sigma_{W X} \\
\Sigma_{X W} & \Sigma_{X X}
\end{array}\right]-\left[\begin{array}{c}
\Sigma_{W W_{0}} \\
\Sigma_{X W_{0}}
\end{array}\right] \Sigma_{W_{0} W_{0}}^{-1}\left(\Sigma_{W_{0} W} \Sigma_{W_{0} X}\right) .
\end{aligned}
$$

Evaluating (5.4) we get the conditional expectations of $W(u, t)$ and $X(u, t)$ given a space maximum at $\mathbf{0}$ with height and curvature $A=a, Z=z$,

$$
\left.\begin{array}{c}
m_{w}(u, t \mid a, z)=\frac{a\left(m_{04} r^{w w}(u, t)+m_{02} r^{w_{u u} w}(u, t)\right)+z\left(m_{02} r^{w w}(u, t)+m_{0} r^{w_{u u} w}(u, t)\right)}{m_{0} m_{04}-m_{02}^{2}}, \\
m_{x}(u, t \mid a, z)=\frac{a\left(m_{04} r^{w x}(u, t)+m_{02} r^{w_{u u} x}(u, t)\right)+z\left(m_{02} r^{w x}(u, t)+m_{0} r^{w_{u u} x}(u, t)\right)}{m_{0} m_{04}-m_{02}^{2}}
\end{array}\right\}
$$

In (5.5) the off-diagonal elements in $C_{\mathcal{W W}}, C_{\mathcal{W X}}, C_{\mathcal{X X}}$ contain the conditional auto-covariance and cross-covariance functions for the normal residual processes. In the following, explicit expressions the time arguments $s, t$ can be changed to space arguments. For covariance functions in space-time one can use the notation $p_{j}=\left(u_{j}, t_{j}\right), j=1,2$, 


\section{G. Lindgren and M. Prevosto}

separated by $\boldsymbol{p}=\boldsymbol{p}_{2}-\boldsymbol{p}_{1}$ as in (5.3). The expressions in time only are

$$
\begin{aligned}
C^{w}(s, t)= & \operatorname{Cov}\left(\Delta^{w}(s), \Delta^{w}(t)\right)=r^{w}(t-s) \\
& -\frac{m_{0} r^{w_{u u} w}(s) r^{w_{u u} w}(t)+m_{02} r^{w}(s) r^{w_{u u} w}(t)}{m_{0} m_{04}-m_{02}^{2}} \\
& -\frac{m_{02} r^{w_{u u} w}(s) r^{w}(t)+m_{04} r^{w}(s) r^{w}(t)}{m_{0} m_{04}-m_{02}^{2}}-\frac{r^{w_{u} w}(s) r^{w_{u} w}(t)}{m_{02}} \\
C^{x}(s, t)= & \operatorname{Cov}\left(\Delta^{x}(s), \Delta^{x}(t)\right)=r^{x}(t-s) \\
& -\frac{m_{0} r^{w_{u u} x}(s) r^{w_{u u} x}(t)+m_{02} r^{w x}(s) r^{w_{u u} x}(t)}{m_{0} m_{04}-m_{02}^{2}} \\
& -\frac{m_{02} r^{w_{u u} x}(s) r^{w x}(t)+m_{04} r^{w x}(s) r^{w x}(t)}{m_{0} m_{04}-m_{02}^{2}}-\frac{r^{w_{u} x}(s) r^{w_{u} x}(t)}{m_{02}} \\
C^{w x}(s, t)= & \operatorname{Cov}\left(\Delta^{w}(s), \Delta^{x}(t)\right)=r^{w x}(t-s) \\
& -\frac{m_{0} r^{w_{u u} w}(s) r^{w_{u u} x}(t)+m_{02} r^{w}(s) r^{w_{u u} x}(t)}{m_{0} m_{4}-m_{2}^{2}} \\
& -\frac{m_{02} r^{w_{u} w}(s) r^{w x}(t)+m_{04} r^{w}(s) r^{w x}(t)}{m_{0} m_{4}-m_{2}^{2}}-\frac{\left.r^{w_{u} w}(s) r^{w_{u} x}(t)\right)}{m_{02}}
\end{aligned}
$$

One should be aware that there are four residual processes involved in the model: $\Delta^{w}(u)$ and $\Delta^{x}(u)$ as space functions, and $\Delta^{w}(t)$ and $\Delta^{x}(t)$ as processes in time, and they are all dependent on each other.

\subsubsection{Explicit Slepian models for the Gaussian components}

We now formulate Slepian models for the Gaussian components conditioned on a local space maximum in $W(u, 0)$ at $u=0$, and from these we describe the Lagrangian wave shape and the corresponding particle orbit. Note that all $\Delta$-processes are correlated through (5.9). The Slepian models for the components are, with the distribution of $A, Z$ given by (4.9) or, when $A$ is fixed, by (4.10)

$$
\begin{aligned}
& \text { Wave models : } \mathcal{W}(u, 0)=m_{w}(u, 0 \mid A, Z)+\Delta^{w}(u, 0) \\
& =A \alpha^{w}(u, 0)+Z \beta^{w}(u, 0)+\Delta^{w}(u, 0), \\
& \mathcal{X}(u, 0)=m_{x}(u, 0 \mid A, Z)+\Delta^{x}(u, 0) \\
& =A \alpha^{x}(u, 0)+Z \beta^{x}(u, 0)+\Delta^{x}(u, 0), \\
& \text { Orbit models : } \quad \mathcal{W}(0, t)=m_{w}(0, t \mid A, Z)+\Delta^{w}(0, t) \\
& =A \alpha^{w}(0, t)+Z \beta^{w}(0, t)+\Delta^{w}(0, t), \\
& \mathcal{X}(0, t)=m_{x}(0, t \mid A, Z)+\Delta^{x}(0, t) \\
& =A \alpha^{x}(0, t)+Z \beta^{x}(0, t)+\Delta^{x}(0, t) \text {. }
\end{aligned}
$$


We note that with $\rho=1 / \tanh (\kappa h)$,

$$
\left.\begin{array}{c}
m_{w}(0,0 \mid a, z)=a, \\
m_{x}(0,0 \mid a, z)=E(X(0,0))=0, \\
C^{w}(0,0)=V\left(\Delta^{w}(0,0)\right)=0, \\
C^{w x}(0,0)=\operatorname{Cov}\left(\Delta^{w}(0,0), \Delta^{x}(0,0)\right)=0, \\
C^{x}(0,0)=V\left(\Delta^{x}(0,0)\right)=\int \rho^{2} S(\omega) \mathrm{d} \omega-\left\{\int \rho \kappa S(\omega) \mathrm{d} \omega\right\}^{2} / m_{02} .
\end{array}\right\}
$$

This means that, at the maximum, the Slepian model for the wave is exactly equal to the height of the maximum, as it should be. The average displacement there is also zero, but randomly normal with non-zero variance, (5.11).

For easy reference we state the functional forms of the Slepian models, leaving out the redundant $t=0$ and $u=0$, respectively,

$$
\left.\begin{array}{c}
\mathcal{W}(u)=A \frac{m_{04} r^{w w}(u)+m_{02} r^{w_{u u} w}(u)}{m_{0} m_{04}-m_{02}^{2}}+Z \frac{\left.m_{02} r^{w w}(u)+m_{0} r^{w_{u u} w}(u)\right)}{m_{0} m_{04}-m_{02}^{2}}+\Delta^{w}(u), \\
\mathcal{X}(u)=A \frac{m_{04} r^{w x}(u)+m_{02} r^{w_{u u} x}(u)}{m_{0} m_{04}-m_{02}^{2}}+Z \frac{\left.m_{02} r^{w x}(u)+m_{0} r^{w_{u u} x}(u)\right)}{m_{0} m_{04}-m_{02}^{2}}+\Delta^{x}(u), \\
\mathcal{W}(t)=A \frac{m_{04} r^{w w}(t)+m_{02} r^{w_{u u} w}(t)}{m_{0} m_{04}-m_{02}^{2}}+Z \frac{\left.m_{02} r^{w w}(t)+m_{0} r^{w_{u u} w}(t)\right)}{m_{0} m_{04}-m_{02}^{2}}+\Delta^{w}(t), \\
\mathcal{X}(t)=A \frac{m_{04} r^{w x}(t)+m_{02} r^{w_{u u} x}(t)}{m_{0} m_{04}-m_{02}^{2}}+Z \frac{\left.m_{02} r^{w x}(t)+m_{0} r^{w_{u u} x}(t)\right)}{m_{0} m_{04}-m_{02}^{2}}+\Delta^{x}(t)
\end{array}\right\}
$$

The formulas are valid both when $A, Z$ are jointly random with density (4.9) and when $A=a$ is fixed and $Z$ is random with conditional density (4.10).

\section{Results: Gaussian Slepian models - wave shape and orbits}

We illustrate the theory on the same orbital spectra as are used in (Lindgren \& Prevosto 2020). The spectrum J20 is a narrow JONSWAP spectrum with significant wave height $H_{s}=4.5 \mathrm{~m}$, peak period $T_{p}=10 \mathrm{~s}$ and $\gamma=20$. The mean max-max wavelength is $40.4 \mathrm{~m}$ and the mean zero crossing wavelength is $112 \mathrm{~m}$. The spectrum PM is a Pierson-Moskowitz wind-sea spectrum with $H_{s}=4.5 \mathrm{~m}, T_{p}=10 \mathrm{~s}$. Its $\max -\mathrm{max}$ and mean zero crossing wavelengths are $16.1 \mathrm{~m}$ and $71 \mathrm{~m}$, respectively. In the simulations the spectra are frequency truncated, the J20 spectrum at $2 \mathrm{rad} \mathrm{s}^{-1}$ and the PM spectrum at $3 \mathrm{rad} \mathrm{s}^{-1}$.

\subsection{Slepian models in space - wave shape}

Figure 3 shows 20 samples of the Slepian processes (5.12) for the J20 model on infinite depth conditioned on a local maximum in $W(u, 0)$ with height $4 \mathrm{~m}$. Panel $(a)$ shows realisations of the Gaussian wave form as a function of $u$ in an interval extending $50 \mathrm{~m}$ around the maximum. The red curves are the regression parts, i.e. the expected Slepian curves (5.6) while the blue curves include the normal residuals around the regression, as in (5.12). Panel $(b)$ shows the horizontal displacement as a function of its original location 


\section{G. Lindgren and M. Prevosto}
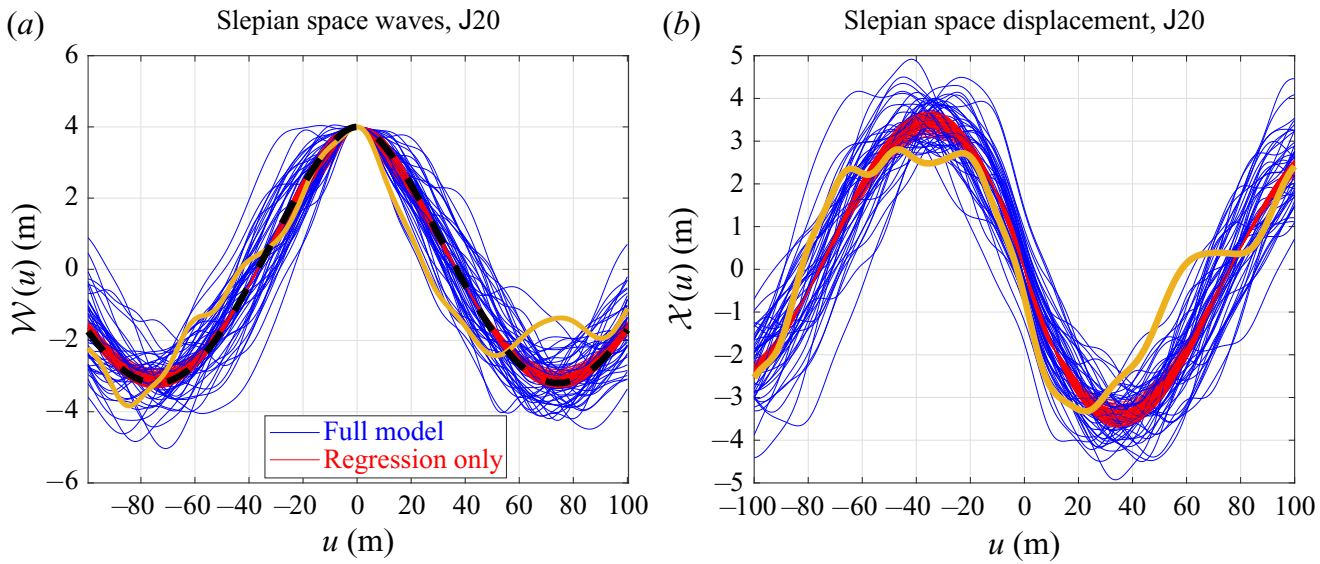

Figure 3. Slepian model realisations of Gaussian wave shape $\mathcal{W}(u)(a)$ and horizontal displacement $\mathcal{X}(u)$ (b) according to (5.12) conditioned on a local maximum with height $a=4 \mathrm{~m}$. The narrow band of red curves shows the regression curves depending on the curvature at maximum, the more variable blue curves are full Slepian models including correlated residuals, independent of maximum height and curvature. The thick brown curves in the two panels come from the same realisation. The black dashed curve in the left plot represents a simplified regression, also called the 'New Wave model', i.e. $a r^{w w}(u, 0) / r^{w w}(0,0)$. The spectrum is the JONSWAP spectrum J20.
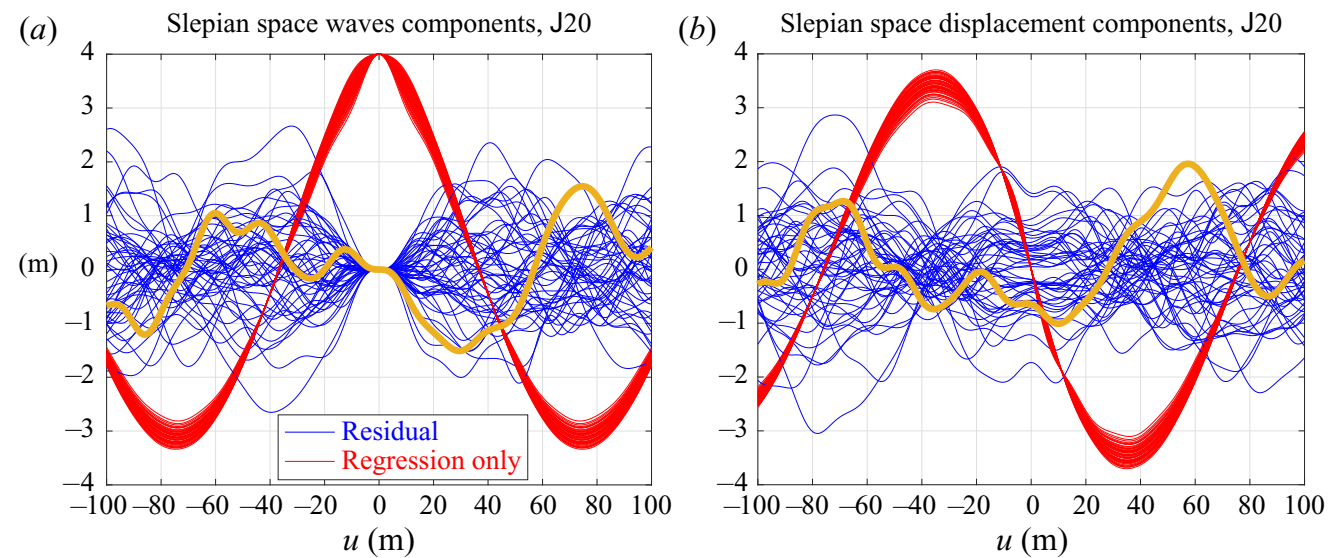

Figure 4. Slepian wave and displacement components for models in figure 3 plotted separately.

relative to the maximum. In the simulations the maximum height was fixed to $a=4 \mathrm{~m}$ and the curvature distribution defined by conditioning in (4.10).

The black dashed curve in figure 3 is the 'New Wave model', defined by Tromans et al. (1991, equation (3)) as $a r^{w w}(u, 0) / r^{w w}(0,0)$, which is the expected (most probable) value conditioned on a stationary point at $u=0$, i.e. $W(0)=a, W^{\prime}(0)=0$, regardless of its curvature. As soon as the curvature is involved, the functional form will be more complicated, (Lindgren 1970, Theorem 3), and (5.6) in the present paper. However, it is easy to see from the conditional density (4.10) that $Z / a$ tends in probability to $-m_{02} / m_{0}$ as $a \rightarrow \infty$. Inserting this limit into the expectation in (5.6) we see that $m_{w}(u, 0 \mid a, z) / a \rightarrow$ $r^{w w}(u, 0) / r^{w w}(0,0)$, i.e. our model is asymptotically equivalent to the Tromans model. 


\section{Orbit orientation in asymmetric waves}
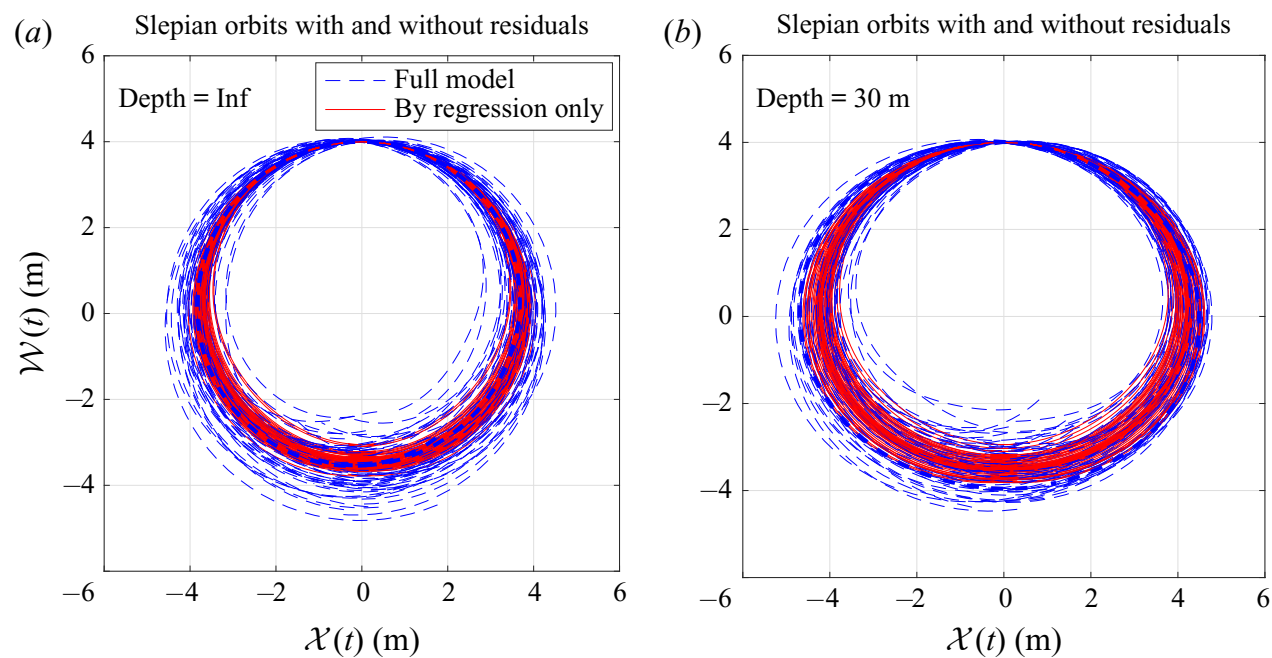

Figure 5. Orbits for the top particles in figure 3 according to models (5.13). (a) Infinite depth; (b) depth 30 $\mathrm{m}$. Red curves are the symmetric regression orbits, blue dashed asymmetric curves include the residuals. The Airy orbital eccentricity of surface particles at water depth $h=30 \mathrm{~m}$ is $\cosh \left(k_{p} h\right) / \sinh \left(k_{p} h\right)=1.14$ for this narrow spectrum with peak frequency $\omega_{p}=0.046 \mathrm{rad} \mathrm{s}^{-1}$. The observed average eccentricity of the simulated regression orbits is 1.12 .

The residuals in figure 3 were simulated from the models (5.7)-(5.9) with dependence between $\Delta^{w}\left(u^{\prime}\right)$ and $\Delta^{x}\left(u^{\prime \prime}\right)$. The residuals were generated by the WAFO-routine rndnormnd from their joint high-dimensional covariance matrix (WAFO-group 2017).

\subsection{Slepian models in time - particle orbits and depth dependence}

For the orbit model we need the Slepian models $\mathcal{X}^{o}(t), \mathcal{W}^{o}(t)$ from (5.13). Figure 5(a) shows orbits from the same model as in figures 3 and 4 . Figure $5(b)$ shows the variation in eccentricity at moderate depth $h=30 \mathrm{~m}$. Here, we have an opportunity to compare the outcomes with classical wave theory that predicts the eccentric elliptic shape of water particles as a function of water depth. The Airy orbital eccentricity of surface particles is $\cosh \left(k_{p} h\right) / \sinh \left(k_{p} h\right)$ where $k_{p}$ is the peak wavenumber and $h$ is the water depth (Kinsman 2002 , p. 137). For the J20 spectrum at depth $h=30 \mathrm{~m}$ this measure takes the value 1.14, very close to the average eccentricity 1.12 of the regression orbits.

\section{Results: Slepian models in the Lagrange model}

From (5.12) and (2.4) we can define a Slepian model for the Lagrange space wave at $t_{0}=0$,

$$
\mathcal{L}^{w}(x)=\mathcal{L}^{w}\left(\mathcal{X}^{w}(u)\right)=\mathcal{W}^{w}(u),
$$

valid in an interval $x^{-}<x<x^{+}$around 0 where $u+\mathcal{X}^{W}(u)$ is strictly increasing. The model for the top particle orbit is defined directly from (5.13),

$$
\left(\mathcal{X}^{o}(t), \mathcal{W}^{o}(t)\right), \quad-\infty<t<\infty \text {. }
$$

We are now ready to focus on the original goal for this paper: the statistical relation between local wave asymmetry and orbit orientation for the top particle in Lagrange waves. 


\section{G. Lindgren and M. Prevosto}

\subsection{Slepian model around local maxima}

We use the same wave definition as in Lindgren \& Prevosto (2020, §3.1), namely the min-max-min definition and apply the Slepian model.

Consider a realisation of the Slepian model $\mathcal{W}^{w}(u)$ with a maximum of height $a$ at $u=0$. Denote by $u^{-}<0<u^{+}$the locations of the local minima closest to 0 and write $m^{-}=\mathcal{W}^{w}\left(u^{-}\right)$and $m^{+}=\mathcal{W}^{w}\left(u^{+}\right)$for their heights. After the Lagrange space shift by $\mathcal{X}^{w}$ the three extrema are located at $\mathcal{X}^{w}\left(u^{-}\right)<\mathcal{X}^{w}(0)<\mathcal{X}^{w}\left(u^{+}\right)$, with order preserved if $u+\mathcal{X}^{w}(u)$ is strictly increasing in $u$. The wave front and back steepness are then defined by

$$
\left.\begin{array}{c}
s^{+}=\left(a-m^{+}\right) /\left(\mathcal{X}^{w}(0)-\mathcal{X}^{w}\left(u^{+}\right)\right)<0, \\
s^{-}=\left(a-m^{-}\right) /\left(\mathcal{X}^{w}(0)-\mathcal{X}^{w}\left(u^{-}\right)\right)>0 .
\end{array}\right\}
$$

The front-back asymmetry is measured in logarithmic scale as $\Lambda=\log \left(-s^{+} / s^{-}\right)$; waves with positive $\Lambda$ have steep front and less steep back.

We define the orbit of the top particle as a function of $\tau$,

$$
O(\tau)=\left(\mathcal{X}^{o}(\tau), \mathcal{W}^{o}(\tau)\right), \quad-d^{-} \leqslant \tau \leqslant d^{+},
$$

where the interval is chosen so that $\mathcal{W}^{w}(\tau)$ has local minima at $-d^{-}$and $d^{+}$, these minima being the closest to 0 .

\subsection{Particle velocities, wave asymmetry and orbital orientation at local maxima}

As a proxy for the orbital orientation one can fit an ellipse to the trajectory in the interval around the centre and use its orientation as a measure of orbit tilt. This was the approach in Lindgren \& Prevosto $(2020, \S 3.3)$, where the MATLAB routine fit_ellipse was used to find the tilt $\theta_{e}$ of the approximating ellipse as a measure of the orbit orientation. It was suggested in that paper that the velocity vector for the top particle would give a more objective measure of the connection between wave asymmetry and orbit orientation. Intuitively, an upward direction would indicate an upward tendency of the orbit and vice versa, and as a local variable it would not be subject to the wild randomness of the orbit.

For the top particle orbits we observe that the maximum in space, $\mathcal{W}^{w}(0)$, is not a maximum in time. We use the velocities $v_{v}=\mathcal{W}_{t}^{o}(0)$ and $v_{h}=\mathcal{X}_{t}^{o}(0)$ to calculate the velocity direction $\theta_{v}=\operatorname{atan} 2\left(v_{v}, v_{h}\right)$. According to (4.5) $v_{v}$ is normal and independent of $v_{h}$, which in turn is the sum of one normal and one Rayleigh variable; its p.d.f. is given in Fact B.1 in Appendix B.

Figure 2 shows the theoretical joint p.d.f. of $v_{h}, v_{v}$ for all local maxima and it agrees with simulations from 100000 simulated Slepian realisations. As seen, they are centred at a positive average horizontal velocity while the vertical one is symmetric around zero. We now investigate the relation to the wave geometry.

We simulate Slepian processes from JONSWAP (J20) and Pierson-Moskowitz (PM) spectra at different depths and observe the Lagrange wave skewness from (7.3). For the orbit orientation we observe both the velocity direction $\theta_{v}$ of the top particle and the orientation $\theta_{e}$ of the fitted ellipse as measures of orbit tilt. We compare with the results in Lindgren \& Prevosto (2020, figure $7 a-c$ ) where waves and orbits were observed in simulated space series. Figure 6 shows the J20 results from simulated Slepian realisations at infinite depth and depths of $h=40,20 \mathrm{~m}$. As in the cited work we restricted the data to 'major', waves with maximum height exceeding $H_{s} / 8$ and front and back amplitudes greater than $H_{S} / 4$. Figure 7 can be compared to figures 2 and 5 in Lindgren \& Prevosto (2020), showing the corresponding results for the PM spectrum. 


\section{Orbit orientation in asymmetric waves}

(a) Tilt/skew pdf, J20, $h=$ inf

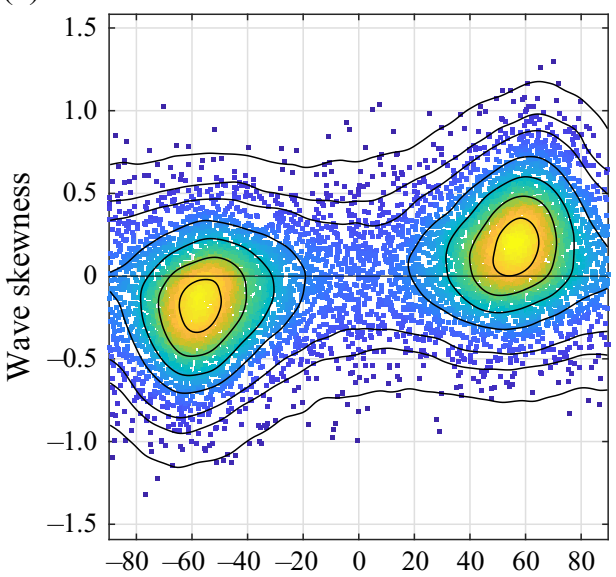

(c)

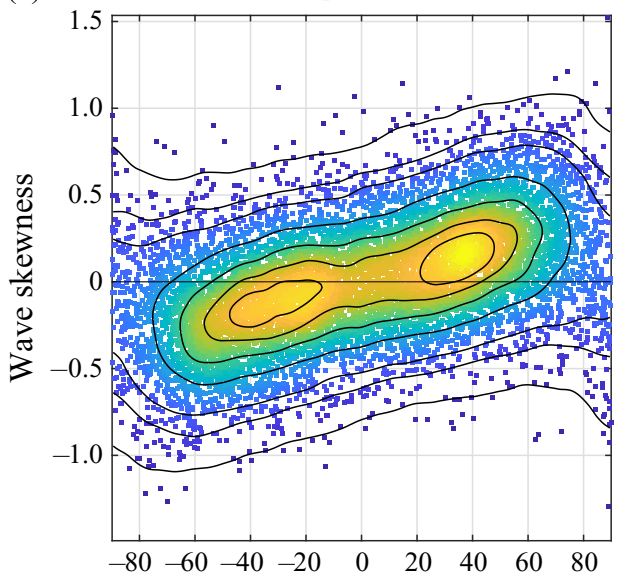

(e) Tilt/skew pdf, $\mathrm{J} 20, h=20$

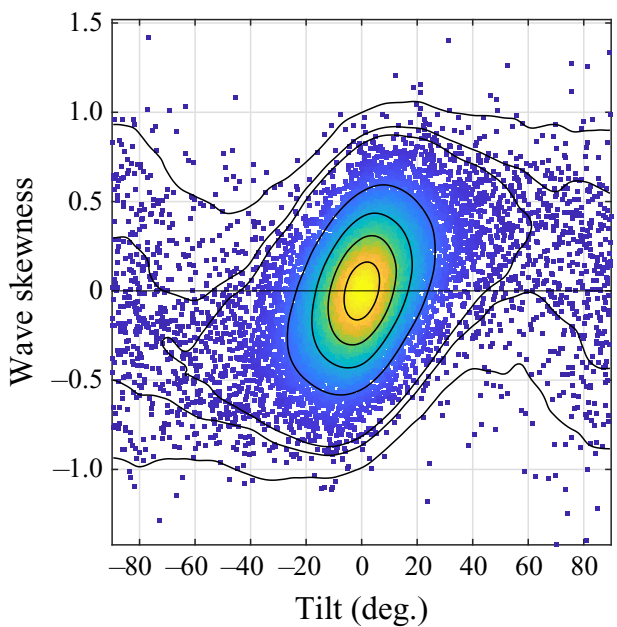

(b)

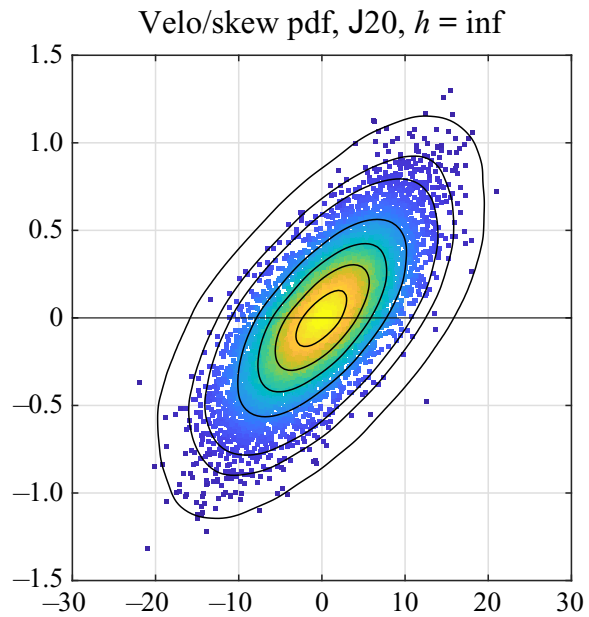

(d)

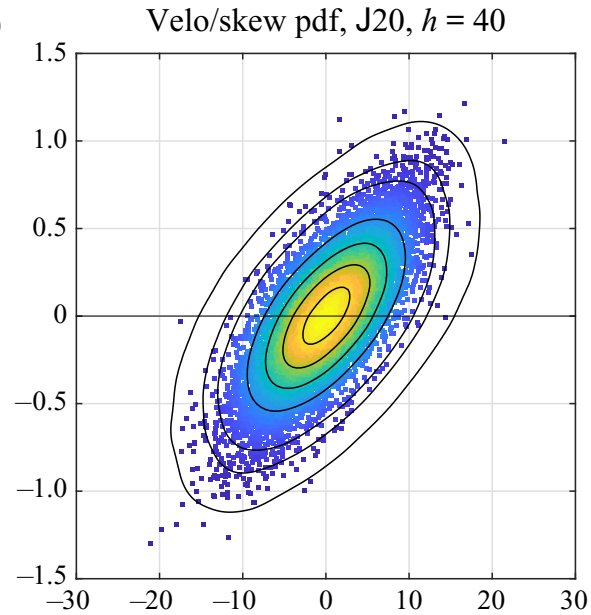

$(f)$

Velo/skew pdf, J20, $h=20$

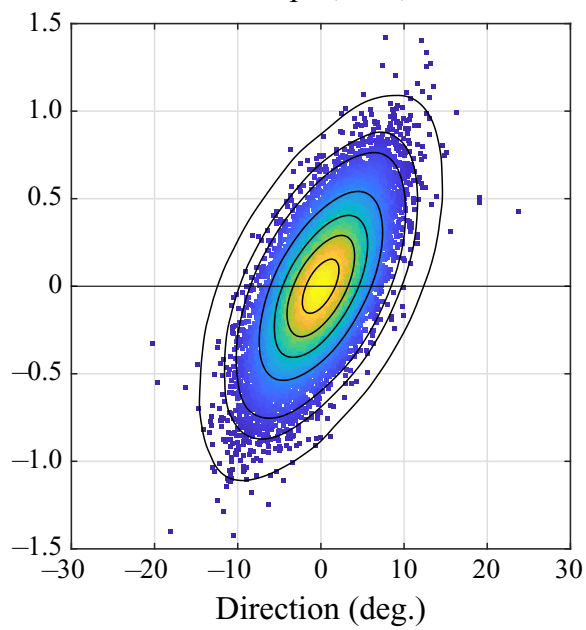

Figure 6. Relation between wave asymmetry and orbit orientation for J20 waves. $(a, c, e)$ Orbit orientation measured by fitted ellipse. $(b, d, f)$ Orbit orientation measured by velocity vector of top particle. Depth from top: infinite, $h=40 \mathrm{~m}, h=20 \mathrm{~m}$. Simulation data with 8000, 9000, 14000 wave/orbit pairs. 


\section{G. Lindgren and M. Prevosto}

(a) Tilt/skew pdf, PM, $h=$ inf

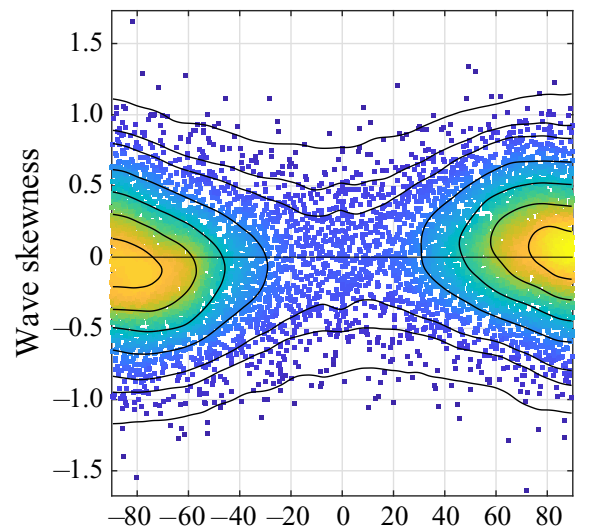

(c)

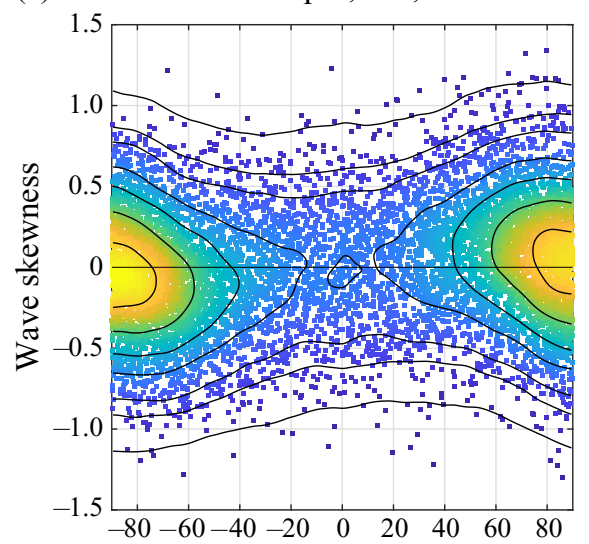

(e) Tilt/skew pdf, PM, $h=20$

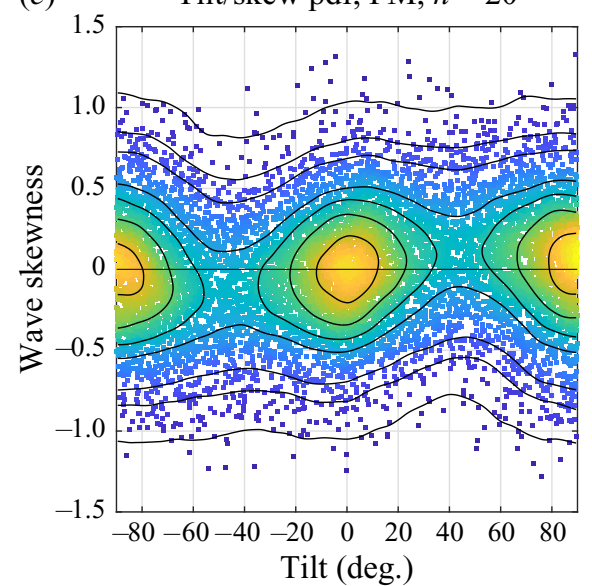

(b) $\quad$ Velo/skew pdf, PM, $h=$ inf

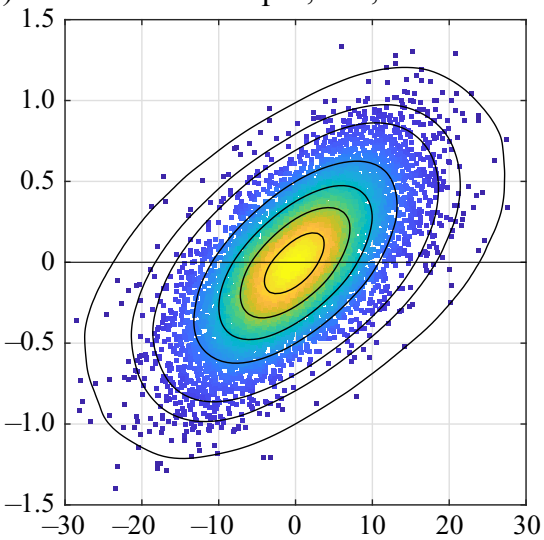

(d) $\quad$ Velo/skew pdf, PM, $h=40$

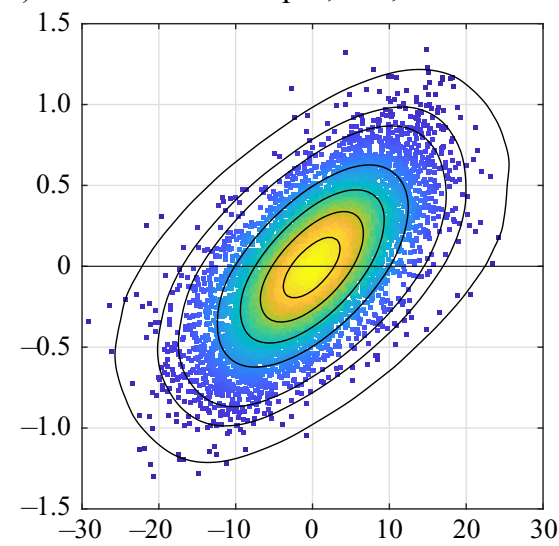

$(f) \quad$ Velo/skew pdf, $\mathrm{PM}, h=20$

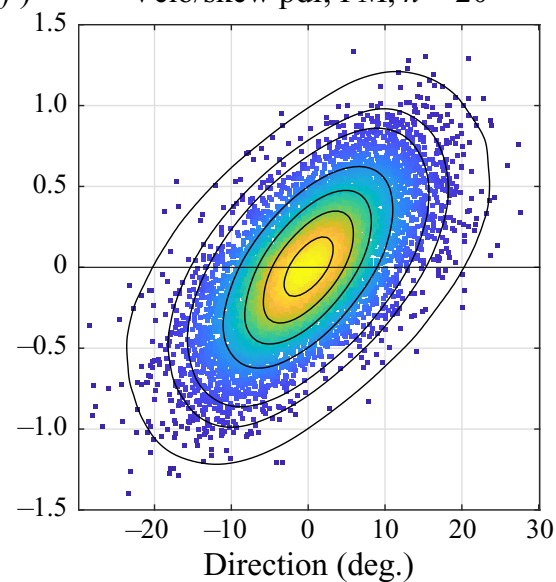

Figure 7. Relation between wave asymmetry and orbit orientation for PM waves. $(a, c, e)$ Orbit orientation measured by fitted ellipse. $(b, d, f)$ Orbit orientation measured by velocity vector of top particle. Depth from top: infinite, $h=40 \mathrm{~m}, h=20 \mathrm{~m}$. Simulation data with approximately 8000, 8000, 9000 wave/orbit pairs. 


\section{Orbit orientation in asymmetric waves}

One can draw the immediate conclusion that both orientation measures have a strong but different statistical relation to the wave asymmetry. Panels $(a, c, e)$ in figures 6 and 7 relate the wave asymmetry to the tilt of the ellipse fitted to the orbit. Panels $(b, d, f)$ show the well-behaved correlation between asymmetry and top velocity orientation. The depth dependence in the tilt measure is much less present in the velocity measure, indicating that the former is partly due to the irregularity of the full orbits, which become more ellipse like with decreasing depth.

\subsection{Slepian models for half waves}

By 'half-crest wave' ('crest wave' for short) we mean a section of the space or time wave that lies between a mean level upcrossing and the following downcrossing. The crest is the largest local maximum of the half-wave. Since the crest also is a local maximum one can use the Slepian model for local maxima to propose a Slepian crest wave, a proposal that is rejected if it is not the maximum between the nearest mean level crossings.

For the Lagrange space waves, the skewness and max particle orbits for half-crest waves are defined as follows, with notations similar to those in $\S 7.1$. A pair of Slepian realisations $\mathcal{W}(u, t), \mathcal{X}(u, t)$ is accepted as part of a Lagrange crest wave if $\mathcal{W}(u, 0)$ is the maximum $\mathcal{W}$ between the nearest upcrossing and downcrossing zeros $u^{-}<0<u^{+}$on either side at time of observation

$$
\left.\begin{array}{c}
A=\mathcal{W}(0,0)>\mathcal{W}(u, 0)>0, \quad u^{-}<u<u^{+}, \\
0=\mathcal{W}\left(u^{-}, 0\right)=\mathcal{W}\left(u^{+}, 0\right) .
\end{array}\right\}
$$

The Lagrange wave steepness is defined by analogy to (7.3),

$$
\left.\begin{array}{l}
s^{+}=A /\left(\mathcal{X}^{w}(0,0)-\mathcal{X}^{w}\left(u^{+}, 0\right)\right)<0, \\
s^{-}=A /\left(\mathcal{X}^{w}(0,0)-\mathcal{X}^{w}\left(u^{-}, 0\right)\right)>0,
\end{array}\right\}
$$

which simplifies the skewness measure to $\Lambda=\log \left(-\left(\mathcal{X}^{w}(0,0)-\mathcal{X}^{w}\left(u^{-}, 0\right)\right) /\right.$ $\left.\left(\mathcal{X}^{w}(0,0)-\mathcal{X}^{w}\left(u^{+}, 0\right)\right)\right)$.

As suggested in Lindgren \& Prevosto $(2020, \S 3)$ the crest wave definition will lead to more chaotic orbits with extra twists and to less characteristic wave skewness measures. We verify this claim and present some of the reasons.

One disturbing random factor is the presence and location of local maxima in the crest wave, besides the crest maximum. The number and allocation of these extra maxima represent a discrete source of disturbing randomness in the skewness measure as can be understood from figure 8 . The skewness distribution will be a discrete mixture of continuous distributions. For example, configuration 1-1 will tend to be centred at 0 while $1-0$ and $0-2$ will be centred near a distinct negative and positive value, respectively.

The probability of extra maxima increases with the length of the crest interval. For the J20 JONSWAP spectrum, used in this example, the distribution of the interval length is strongly bimodal, with one peak near $10 \mathrm{~m}$ and one near $40 \mathrm{~m}$, enhancing the mixture effect; figure 9. For the PM spectrum, the crest interval distribution has a sharp peak for short intervals and a flat part between 30 and $50 \mathrm{~m}$, enough to cause a discrete skewness measure.

The probability of extra maxima in a crest wave is also related to the height of the crest. Small crests have approximately a parabolic shape, (Slepian 1963, §3), with asymptotically equal front and back periods, leading to an asymmetry measure near zero. This introduces an extra disturbance in the relation between wave asymmetry and top 


\section{G. Lindgren and M. Prevosto}

(a)

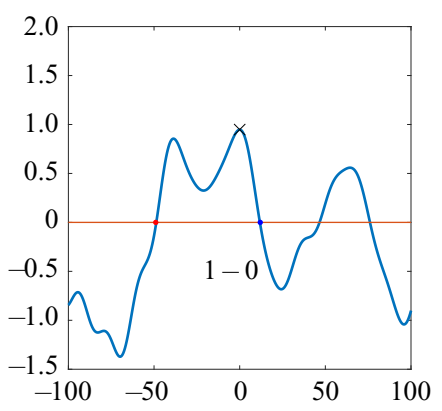

(b)

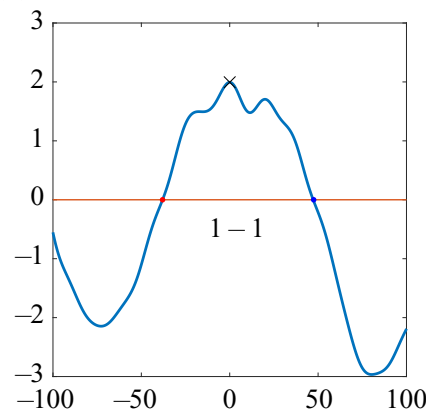

(c)

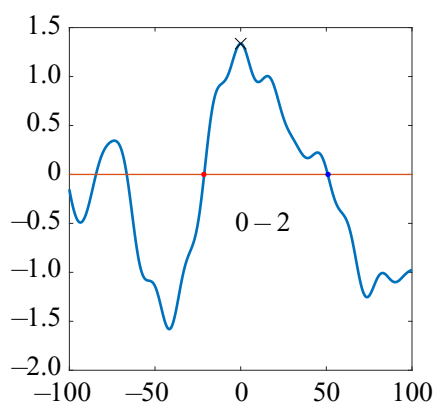

Figure 8. Illustration of the discrete dependence of crest location (marked with $\times$ ) and the number of local maxima in a crest period. The code $x-y$ indicates the number of local maxima to the left and right of the crest, respectively.

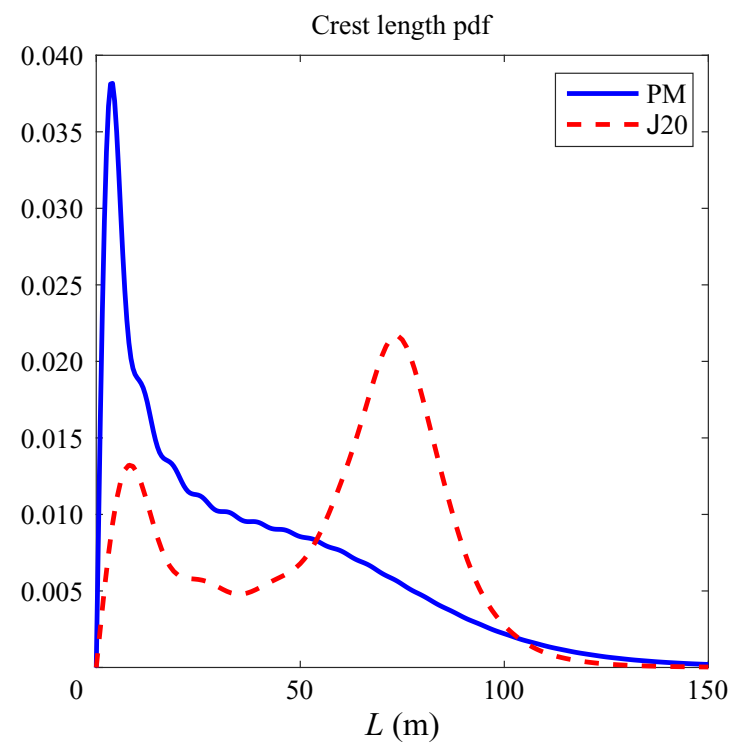

Figure 9. Probability densities of crest wavelength for PM (solid, blue) and J20 (dashed, red) spectra, computed by the WAFO routine spec 2 tpdf, (WAFO-group 2017).

particle direction as is seen in figure 10. The figure shows simulated pairs of top particle directions and wave asymmetry for crest waves with only one local maximum. Blue dots illustrate the distribution with all maxima included, black dots show pairs with maximum above $3 \mathrm{~m}$, and red dots come from small waves with height less than $0.1 \mathrm{~m}$. Simulations from the PM spectrum give the same type of crest height dependence.

REMARK 7.1. Slepian simulation of crest waves can be made by the original simple Slepian model after mean level upcrossings and crest height and location as well as front and back periods observed in the realisations. This method is very fast and it gives the same results for the wave geometry as the method we use here, but it does not give any information on the top particle velocities. 


\section{Orbit orientation in asymmetric waves}

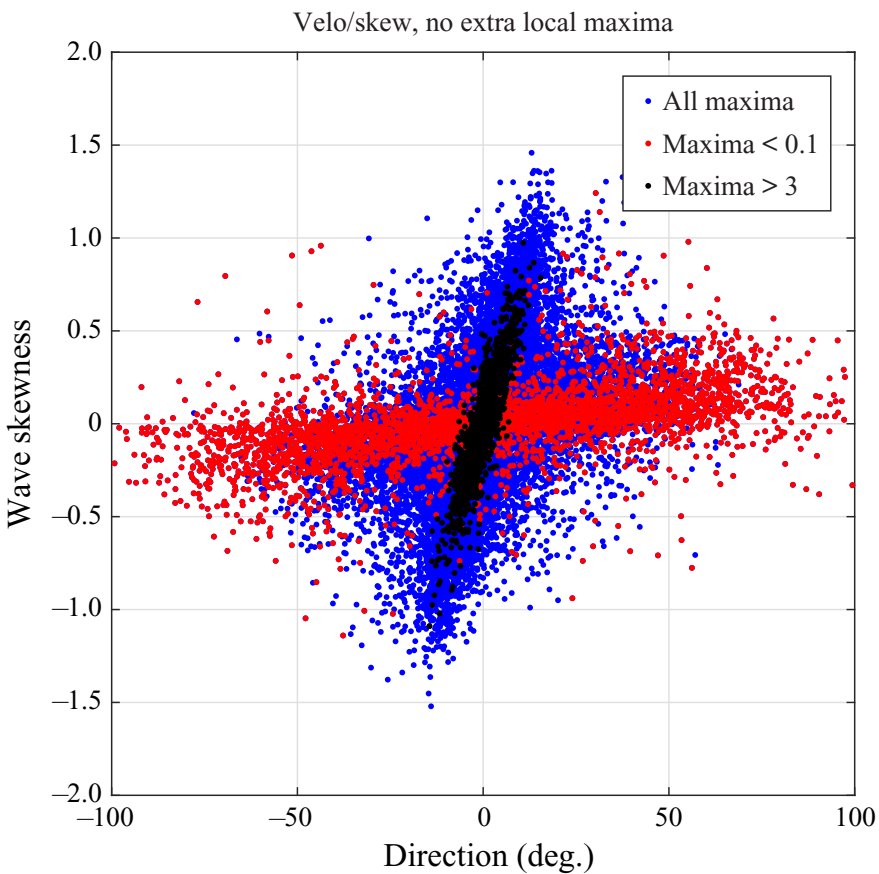

Figure 10. Illustration of the dependence of crest height on the relation between top particle direction and crest wave skewness, J20 spectrum.

\section{Discussion}

Our analysis gives rise to several questions that need to be discussed, and here we address two: the effect of second-order Stokes corrections and the importance of crest height.

Wave crest-trough asymmetry: the Gauss-Lagrange waves exhibit crest-trough asymmetry very similar to that in second- and higher-order Stokes models (Tayfun 1980) but the mechanisms are fundamentally different. In the Stokes model, wave crests become more peaked and troughs are flattened by the addition of frequency interaction terms. In the Gauss-Lagrange model crests and troughs present in the vertical Gaussian component remain crests and troughs after the horizontal displacement and their heights are unaltered. Crests are narrowed and troughs flattened by the displacement.

Wave front-back asymmetry: the focus in this paper is on the relation between the stochastic variation of wave front-back asymmetry and the direction of the crest top particle. A relevant question regards how the derived relation changes when Stokes terms are added to the wave profiles. For the spectra we have used, the standard deviation of the vertical and horizontal effects of the second-order Stokes correction is 5\%-10\% of those of the Gaussian terms and the effect on wave asymmetry is small. For the crest particle velocity the effects are similarly small. For the J20 spectrum the Stokes term causes large but slow horizontal oscillating drifts, the standard deviation of the added horizontal velocity is approximately $10 \%$ of the Gaussian velocity. The vertical velocity is not affected. For the PM spectrum the effects of the Stokes correction are even smaller. The effect on the asymmetry-velocity relations in figures 6 and 7 is small.

Stokes drift: as observed in Lindgren \& Prevosto (2020, § 3.4) the Stokes drift has a very small effect on the wave asymmetry but it has a systematic effect on the horizontal 


\section{G. Lindgren and M. Prevosto}

(a)

Slepian space wave components, J20

(m)

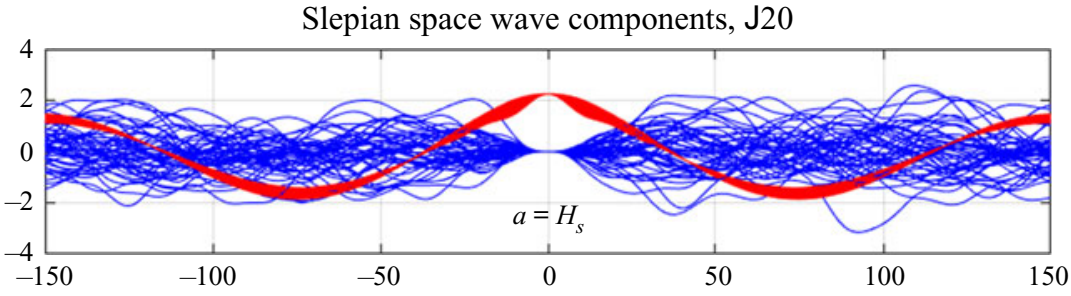

(b)

(m)

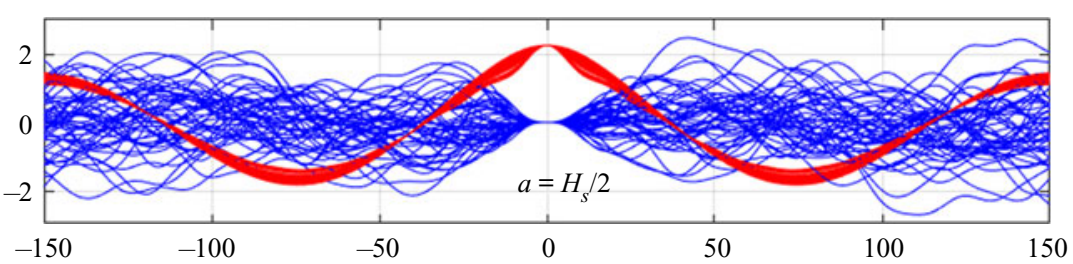

(c)

(m)

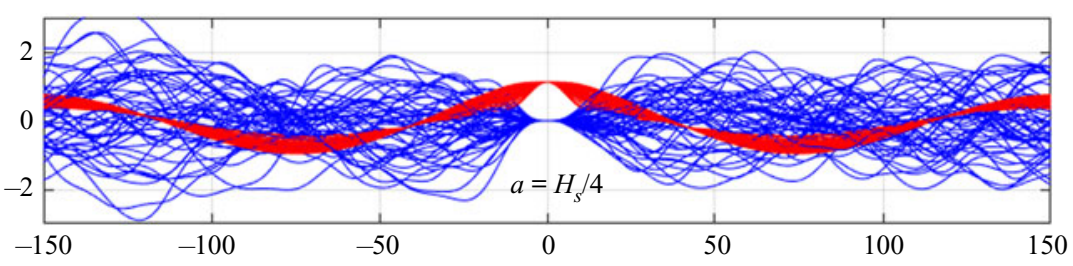

Figure 11. Height dependence illustrating the balance between the variability of regression (red smooth curves) and residual (blue irregular curves) for different crest heights $a$. Sea state is the $\mathbf{J} 20$ model.

particle velocity. The effect depends on the crest height and is of smaller order than the model velocity illustrated in figure 1 .

Dependence on crest height: it is worth noting that the residual process in the Slepian models does not depend on the crest height. Close to the maximum its variability is very small but it increases abruptly with distance from the crest and overshadows the regression term, which is proportional to the maximum height. This is illustrated in figure 11.

\section{Summary and conclusions}

We have shown how a Slepian model can be used for detailed studies of the front-back asymmetry of irregular ocean waves and how wave asymmetry is related to the kinematics of the particle near wave maxima in space. Based on Gaussian field models for the Lagrangian vertical and horizontal particle movements we present explicit expressions for the complete distribution of the local wave process and for the random top particle orbit, as well as for their joint statistical distribution. Examples from two very different wave spectra, one narrow JONSWAP spectrum and one Pierson-Moskowitz wind-wave spectrum, show that there is a stable systematic covariation between the orientation of the top particle movement and the degree of front-back asymmetry. The relation is not sensitive to deviations from the Gaussian assumptions and could be empirically adapted to field observations of particle kinematics.

The Slepian model is a very versatile tool for detailed analysis of crossing related events in a stochastic process or field. It is explicit and very easy and fast to simulate and it lends itself to analysis also of very rare events. In our analysis we derived a Slepian model for individual space waves defined as the part of the surface between two local 
minima with the wave centred at the intermediate local maximum. A companion model is given for the particle orbits, i.e. the horizontal movements in time for the particle located at the wave maximum. Both models include the height and the curvature at the wave maximum as random parameters in a deterministic regression part surrounded by non-stationary Gaussian residual processes. The variability of the residual compared with that of the regression gives a hint of how far away from the wave maximum one can expect to draw reliable conclusions. As it is locally defined, the top particle orientation is a stable measure of orbit orientation and its covariation with wave asymmetry is not affected by the non-local residual variability.

As an alternative to the min-max-min wave definition we also used the Slepian technique to the crest half-wave definition, used in our previous study of the asymmetry-orbit relation, (Lindgren \& Prevosto 2020). We conclude here that the top particle method can give more stable relations to the wave asymmetry than the ellipse tilt method used in the previous paper, but that the presence of extra local maxima disturbs the clear relation. We identified the bi-modality of the crest wavelength distribution as an important factor, complicating the relation between wave asymmetry measure and orbit orientation.

Slepian models have great potential in ocean wave modelling, and we finish with two recent examples where a Slepian formulation might be fruitful.

The first example concerns wave breaking, a highly nonlinear phenomenon. Waves obtained from Gaussian modelling are far from the extreme nonlinear waves that are encountered close to breaking conditions. On the other hand, Gaussian modelling permits one to obtain statistical distributions for extreme combinations of random quantities characteristic for 'real world' nonlinearities derived from deterministic nonlinear wave equations. For example, in this way, Stringari et al. (2021) derive wave breaking probabilities based on a crest particle velocity-speed of local maxima ratio criterion. Applied to classical wave breaking criteria, the new formula compares well with historical methods on recorded data. The second example (Hlophe et al. 2021) concerns wave-to-wave prediction in weakly nonlinear wave fields, where crossing conditioning could be useful.

Acknowledgements. This research received no specific grant from any funding agency, commercial or not-for-profit sectors.

Declaration of interests. The authors report no conflict of interest.

Author ORCIDs.

(1) Georg Lindgren https://orcid.org/0000-0002-1442-4774;

Marc Prevosto https://orcid.org/0000-0001-7706-2873.

\section{Appendix A. Some moments and covariance functions}

We need the following moments and covariance functions for the $W$ - and $X$-fields and their derivatives, for example

$$
W_{u}(0,0)=\frac{\partial W(u, t)}{\partial u}(0,0), \quad W_{u u}(0,0)=\frac{\partial^{2} W(u, t)}{\partial u^{2}}(0,0) .
$$




\section{G. Lindgren and M. Prevosto}

We use a one-sided orbital spectrum $S(\omega), \omega>0$ with notations $\gamma=\kappa u-\omega t$ and $\rho=$ $1 / \tanh (\kappa h)$,

$$
\begin{aligned}
& m_{0}=V(W(0,0))=\int S(\omega) \mathrm{d} \omega, \\
& m_{02}=V\left(W_{u}(0,0)\right)=\int \kappa^{2} S(\omega) \mathrm{d} \omega, \\
& m_{04}=V\left(W_{u u}(0,0)\right)=\int \kappa^{4} S(\omega) \mathrm{d} \omega, \\
& m_{20}=V\left(W_{t}(0,0)\right)=\int \omega^{2} S(\omega) \mathrm{d} \omega \\
& m_{11}=-\operatorname{Cov}\left(W_{u}(0,0), W_{t}(0,0)\right)=\int \omega \kappa S(\omega) \mathrm{d} \omega, \\
& \hat{m}_{0}=V(X(0,0))=\int \rho^{2} S(\omega) \mathrm{d} \omega, \\
& \hat{m}_{10}=\operatorname{Cov}\left(W(0,0), X_{t}(0,0)\right)=\int \rho \omega S(\omega) \mathrm{d} \omega, \\
& \hat{m}_{20}=V\left(X_{t}(0,0)\right)=\int \rho^{2} \omega^{2} S(\omega) \mathrm{d} \omega, \\
& \hat{m}_{12}=-\operatorname{Cov}\left(W_{u u}(0,0), X_{t}(0,0)\right)=\int \rho \omega \kappa^{2} S(\omega) \mathrm{d} \omega, \\
& r^{w w}(u, t)=\operatorname{Cov}(W(0,0), W(u, t))=\int \cos (\gamma) S(\omega) \mathrm{d} \omega, \\
& r^{x x}(u, t)=\operatorname{Cov}(X(0,0), X(u, t))=\int \rho^{2} \cos (\gamma) S(\omega) \mathrm{d} \omega, \\
& r^{w x}(u, t)=\operatorname{Cov}(W(0,0), X(u, t))=-\int \rho \sin (\gamma) S(\omega) \mathrm{d} \omega, \\
& r^{w_{u} w}(u, t)=\operatorname{Cov}\left(W_{u}(0,0), W(u . t)\right)=\int \kappa \sin (\gamma) S(\omega) \mathrm{d} \omega, \\
& r^{w_{u} x}(u, t)=\operatorname{Cov}\left(W_{u}(0,0), X(u, t)\right)=\int \rho \kappa \cos (\gamma) S(\omega) \mathrm{d} \omega, \\
& r^{w_{u u} w}(u, t)=\operatorname{Cov}\left(W_{u u}(0,0), W(u, t)\right)=-\int \kappa^{2} \cos (\gamma) S(\omega) \mathrm{d} \omega, \\
& r^{w_{u u} x}(u, t)=\operatorname{Cov}\left(W_{u u}(0,0), X(u, t)\right)=\int \rho \kappa^{2} \sin (\gamma) S(\omega) \mathrm{d} \omega .
\end{aligned}
$$

\section{Appendix B. Rayleigh shifted normal distributions}

A Slepian process in Gaussian models normally consists of the sum of a Rayleigh variable and an independent normal variable or process. We present here the general expression for the p.d.f. of such a sum of variables. The formula for a single normal variable was 
originally given by Rice $(1945, \S 3.6)$, and it reads in general form (Lindgren 2013, equation (8.37)) as in the following fact B.1.

FACT .1. The probability density for the sum of a mean zero normal variable and an independent Rayleigh variable, $Z=\sigma_{1} N+\sigma_{2} R$, with standard $N \sim N(0,1), R \sim \operatorname{Ray}(1)$, $i$, with $\Phi$ the standard normal distribution function,

$$
\begin{aligned}
f_{Z}(z)= & \frac{\sigma_{2} z}{\left(\sigma_{1}^{2}+\sigma_{2}^{2}\right)^{3 / 2}} \exp \left(-\frac{z^{2}}{2\left(\sigma_{1}^{2}+\sigma_{2}^{2}\right)}\right) \Phi\left(\frac{\sigma_{2} z}{\sigma_{1} \sqrt{\sigma_{1}^{2}+\sigma_{2}^{2}}}\right) \\
& +\frac{\sigma_{1}}{\sqrt{2 \pi}\left(\sigma_{1}^{2}+\sigma_{2}^{2}\right)} \exp \left(-\frac{z^{2}}{2 \sigma_{1}^{2}}\right) .
\end{aligned}
$$

FACT .2. Let $X=\left[\begin{array}{l}X_{1} \\ X_{2}\end{array}\right]$ have a bivariate normal distribution with mean zero and covariance matrix $\Sigma$ and let $R$ be a standard Rayleigh variable, independent of $X$. Define the Rayleigh translated normal variable

$$
Y=X+R\left[\begin{array}{l}
1 \\
1
\end{array}\right]
$$

with density as the convolution

$$
f_{Y}(y)=\int_{0}^{\infty} r e^{-r^{2} / 2} \times \frac{1}{2 \pi \sqrt{\operatorname{det} \Sigma}} \exp \left\{-\frac{1}{2}(y-r)^{\prime} A(y-r)\right\} \mathrm{d} r
$$

where $A=\Sigma^{-1}=\left[\begin{array}{ll}a_{11} & a_{12} \\ a_{12} & a_{22}\end{array}\right]$. Expanding the quadratic form

$$
\begin{aligned}
(\boldsymbol{y}-\boldsymbol{r})^{\prime} A(\boldsymbol{y}-\boldsymbol{r})= & a_{11} y_{1}^{2}+2 a_{12} y_{1} y_{2}+a_{22} y_{2}^{2} \\
& -2 r\left(a_{11} y_{1}+a_{12}\left(y_{1}+y_{2}\right)+a_{22} y_{2}\right)+r^{2}\left(a_{11}+2 a_{12}+a_{22}\right) \\
= & \alpha\left(y_{1}, y_{2}\right)-2 \beta\left(y_{1}, y_{2}\right) r+\gamma r^{2}=\alpha-\beta^{2} / \gamma+\gamma(r-\beta / \gamma)^{2},
\end{aligned}
$$

we get

$$
\begin{aligned}
f_{Y}(y)= & \int_{0}^{\infty} r \exp \left\{-r^{2} / 2\right\} \times \frac{\sqrt{\gamma}}{\sqrt{2 \pi}} \exp \left\{-\frac{\gamma}{2}(\beta / \gamma-r)^{2}\right\} \mathrm{d} r \\
& \times \frac{1}{\sqrt{2 \pi \gamma \operatorname{det} \Sigma}} \exp \left\{-\frac{1}{2}\left(\alpha-\beta^{2} / \gamma\right)\right\} .
\end{aligned}
$$

The first factor is the convolution of a Rayleigh density and a normal density with mean 0 and variance $1 / \gamma$, i.e. it is equal to the p.d.f. (B1) with $\sigma_{2}=1$ of $R+N / \sqrt{\gamma}$ evaluated at $\beta / \gamma$, while the second factor is explicit. To apply the formula to the distribution in (4.5) we just normalise the relevant equations.

\section{REFERENCES}

ÅBerg, S. 2007 Wave intensities and slopes in Lagrangian seas. Adv. Appl. Prob. 39, 1020-1035. ADLER, R. 1981 The Geometry of Random Fields. John Wiley and Sons.

AzAïs, J.-M. \& Chassan, M. 2020 Discretization error for the maximum of a Gaussian field. Stoch. Proc. Appl. 130 (2), 545-559. 


\section{G. Lindgren and M. Prevosto}

Baxevani, A., PodgóRski, K. \& Rychlik, I. 2003 Velocities for moving random surfaces. Prob. Engng Mech. 18, 251-271.

BaxeVAni, A. \& Wilson, R. 2018 Prediction of catastrophes in space over time. Extremes 21, 601-628.

Blanco-Pillado, J., Sousa, K. \& Urkiola, M. 2020 Slepian models for Gaussian random landscapes. J. High Energy Phys. 2020, 142.

BoccotTI, P. 1982 On ocean waves with high crests. Meccanica 17, 16-23.

Bоссотті, P. 1983 Some new results on statistical properties of wind waves. Appl. Ocean Res. 5, 134-140.

Bоссотті, P. 1984 Sea waves and quasi-determinism of rare events in rare events in random processes. Atti Accad. Naz. Lincei 76, 119-127.

Bоссотті, P. 2000 Wave Mechanics for Ocean Enineering. Elsevier Science.

Boccotti, P. 2015 Wave Mechanics and Wave Loads on Marine Structures. Elsevier Science.

van den Bremer, T.S., Whittaker, C., Calvert, R., Raby, A. \& TAylor, P.H. 2019 Experimental study of particle trajectories below deep-water surface gravity wave groups. J. Fluid Mech. 879, 168-186.

Calvert, R., Whittaker, C., Raby, A., Taylor, P.H., Borthwick, A.G.L. \& van Den Bremer, T.S. 2019 Laboratory study of the wave-induced mean flow and set-down in unidirectional surface gravity wave packets on finite water depth. Phys. Rev. Fluids 4, 114801.

Chen, Y.-Y., Hsu, H.-C. \& CHEN, G.-Y. 2010 Lagrangian experiment and solution for irrotational finite-amplitude progressive gravity waves at uniform depth. Fluid Dyn. Res. 42, 045511.

Chen, Y.-Y., LI, M.-S., Hsu, H.-C. \& NG, C.-O. 2012 Theoretical and experimental study of particle trajectories for nonlinear water waves propagating on a sloping bottom. Phil. Trans. R. Soc. Lond. A 370, $1543-1571$.

DiBenedeTto, M.H. 2020 Non-breaking wave effects on byoyant particle distributions. Front. Mar. Sci. 7, 148.

Dimentberg, M., Iourtchenko, D. \& NAess, A. 2006 Short-term structural instability under random dynamic loads. J. Engng Mech. ASCE 132, 1269-1272.

Ditlevsen, O. 1985 Survey on applications of Slepian model processes in structural reliability. In Proceedings of the 4th International Conference on Structural Safety and Reliability, ICOSSAR'85 (ed. I Konishi, A. Ang \& M. Shinozuka), vol. 1, pp. 241-150. IASSAR.

Ditlevsen, O. \& TARP-Johansen, N.J. 1999 Slepian modelling as a computational method in random vibration analysis of hysteretic structures. In Computational Stochastic Mechanics (ed. P.D. Spanos). Balkema.

FEAU, C. 2008 Probabilistic response of an elastic perfectly plastic oscillator under Gaussian white noise. Prob. Engng Mech. 23, 36-44.

GADRICH, T. \& ADLER, R.J. 1993 Slepian models for non-stationary Gaussian processes. J. Appl. Prob. 30 (1), 98-111.

Gerstner, F.J. 1809 Theorie der Wellen. Ann. Phys. 32, 412-445.

GrigoriU, M. 2020 First passage times for Gaussian processes by Slepian models. Prob. Engng Mech. 61, 103086.

GRUE, J. \& Jensen, A. 2012 Orbital velocity and breaking in steep random gravity waves. J. Geophys. Res. 117, C07013.

Grue, J. \& KolaAs, J. 2017 Experimental particle paths and drift velocity in steep waves at finite water depth. J. Fluid Mech. 810, R1.

Guérin, C.-A., Desmars, N., Grilli, S.T., Ducrozet, G., Perignon, Y. \& Ferrant, P. 2019 An improved Lagrangian model for the time evolution of nonlinear surface waves. J. Fluid Mech. 876, 527-552.

Hlophe, T., Wolgamot, H., Kurniawan, A., Taylor, P.H. \& Orszaghova, J. 2021 Fast wave-by-wave prediction of weakly nonlinear unidirectional wave fields. Appl. Ocean Res. 112, 102695.

Kinsman, B. 2002 Wind Waves: Their Generation and Propagation on the Ocean Surface. Dover.

Lazarov, B. \& Ditlevsen, O. 2005 Slepian simulation of distributions of plastic displacements of earthquake exicted shear frames with a large number of stories. Prob. Engng Mech. 20, 251-262.

Leadbetter, M.R., Lindgren, G. \& Rootzén, H. 1983 Extremes and Related Properties of Random Sequences and Processes. Springer.

LindgRen, G. 1970 Some properties of a normal process near a local maximum. Ann. Math. Statist. 41, 1870-1883.

LiNDGREN, G. 1972 Local maxima of Gaussian fields. Ark. Mat. 10, 195-218.

Lindgren, G. 1977 Functional limits of empirical distributions in crossing theory. Stoch. Proc. Appl. 5, $143-149$.

LindGren, G. 2006 Slepian models for the stochastic shape of individual Lagrange sea waves. Adv. Appl. Prob. 38, 430-450. 


\section{Orbit orientation in asymmetric waves}

LiNDGREN, G. 2012 A detailed statistical representation of the local structure of optical vortices in random wavefields. J. Opt. A 14, 035704.

Lindgren, G. 2013 Stationary Stochastic Processes - Theory and Applications. CRC Press.

LINDGREN, G. 2015 Horseshoe-like patterns in first-order 3D random Gauss-Lagrange waves with directional spreading. Wave Random Complex 25, 729-745.

Lindgren, G. \& PREvosto, M. 2020 Wave asymmetry and particle orbits in irregular wave models. J. Fluid Mech. 905, A27.

LindGREN, G. \& RYCHLIK, I. 1982 Wave characteristics distributions for Gaussian waves - wave-length, amplitude and steepness. Ocean Engng 9, 411-432.

LiNDGREN, G. \& RYCHLIK, I. 1991 Slepian models and regression approximations in crossing and extreme value theory. Intl Stat. Rev. 59, 195-225.

VAN DE LINDT, J.W. \& NIEDZWECKI, J.M 2005 Structural response and reliability estimates: Slepian model approach. J. Struct. Engng 131, 1620-1628.

Miche, M. 1944 Mouvements ondulatoires de la mer on profondeur constante ou décroissante. Forme limit de la houle lors de son déferlement. Application aux digues marines. Ann. Ponts Chassées 114, 25-78.

NiedzWeCKI, J.M. \& SANDT, E.W. 1999 Characterizing the excitation and response of a spar buoy in random seas. In Proceedings Oceans '99 MTS/IEEE Riding the Crests into the 21st Century, Seattle, WA, pp. 104-106. Marine Technology Soc.

Nouguier, F., Chapron, B. \& GuÉRIn, C.-A. 2015 Second-order Lagrangian description of tri-dimensional gravity wave interactions. J. Fluid Mech. 772, 165-196.

Nouguier, F., Guérin, C.-A. \& Chapron, B. 2009 'Choppy wave' model for nonlinear gravity waves. J. Geophys. Res. 114, C09012.

Phillips, O.M., Gu, D. \& Donelan, M. 1993a Expected structure of extreme waves in a Gaussian sea. Part I: theory and SWADE buoy experiments. J. Phys. Oceanogr. 23, 992-1000.

Phillips, O.M., Gu, D. \& WAlSh, E.J. $1993 b$ Expected structure of extreme waves in a Gaussian sea. Part II: SWADE scanning radar altimeter measurements. J. Phys. Oceanogr. 23, 2297-2309.

Pierson, W.J. 1961 Models of Random Seas Based on the Lagrangian Equations of Motion. New York University, College of Engineering, Research Division, Dept. of Meteorology and Oceanography.

PODGÓRSKI, K., RYCHLIK, I. \& WALLIN, J. 2015 Slepian noise approach for Gaussian and Laplace moving average processes. Extremes 18, 665-695.

RANDRup-Thomsen, S. \& Ditlevsen, O. 1997 One-floor building as elasto-plastic oscillator subject to and interacting with Gaussian base motion. Prob. Engng Mech. 12, 49-56.

RICE, S.O. 1945 Mathematical analysis of random noise. Bell. Syst. Tech. J. 23, 46-156.

Romero, L. \& Melville, W.K. 2010 Airborne observations of fetch-limited waves in the Gulf of Tehuantepec. J. Phys. Oceanogr. 40, 441-465.

RYCHLIK, I. 1987 Regression approximations of wavelength and amplitude distributions. Adv. Appl. Prob. 19, 396-430.

SlePIAN, D. 1963 On the zeros of Gaussian noise. In Proceedings Brown Symposium Time Ser. Anal. 1962 (ed. M. Rosenblatt). John Wiley \& Sons.

SoвCZYк, K. 1993 Stochastic modelling of fatigue: methodological background. In Stochastic Approach to Fatigue: Experiments, Modelling and Reliability (ed. K. Sobczyk). Springer.

Stringari, C.E., Prevosto, M., Filipot, J.-F., Leckler, F. \& Guimarães, P.V. 2021 A new probabilistic wave breaking model for dominant wind-sea waves based on the Gaussian field theory. J. Geophys. Res.: Oceans 126, e2020JC016943.

SZAJNOWSKI, W.J. 1996 A fast time-domain estimator of the bandwidth of a Gaussian process. IEEE Signal Proc. Lett. 3, 182-183.

TAYFun, M.A. 1980 Narrow-band nonlinear sea waves. J. Geophys. Res. 85, 1548-1552.

Tromans, P.P., Anaturk, A.R. \& Hagemeijer, P. 1991 A new model for the kinematics of large ocean waves - application as a design wave. In Proceedings of the 1st International Offshore Polar Engineering Conference (ed. J.S. Chung), ISOPE, vol. III, pp. 64-71. Edinburgh, corrected version 2016.

WAFO-GROUP 2017 Wafo - a Matlab toolbox for analysis of random waves and loads. https://github.com/ wafo-project.

Whittaker, C.N., Raby, A.C., Fitzgerald, C.J. \& Taylor, P.H. 2016 The average shape of large waves in the coastal zone. Coast. Engng 114, 253-264.

Winterstein, S.R., Torhaug, R. \& KumaR, S. 1998 Design seastates for extreme response of jackup structures. J. Offshore Mech. Arctic Engng 120, 103-108. 